\title{
Component-based model to predict aerodynamic noise from high-speed train pantographs.
}

\author{
E. Latorre Iglesias*, D.J. Thompson, M.G. Smith \\ Institute of Sound and Vibration Research, University of Southampton, Southampton, SO17 1BJ, United Kingdom
}

\begin{abstract}
At typical speeds of modern high-speed trains the aerodynamic noise produced by the airflow over the pantograph is a significant source of noise. Although numerical models can be used to predict this they are still very computationally intensive. A semi-empirical component-based prediction model is proposed to predict the aerodynamic noise from train pantographs. The pantograph is approximated as an assembly of cylinders with particular cross-sections. An empirical database is used to obtain the coefficients of the model to account for various factors: incident flow speed, diameter, cross-sectional shape, yaw angle, rounded edges, length-to-width ratio, incoming turbulence and directivity. The overall noise from the pantograph is obtained as the incoherent sum of the predicted noise from the different pantograph struts. The model is validated using available wind tunnel noise measurements of two fullsize pantographs. The results show the potential of the semi-empirical model to be used as a rapid tool to predict aerodynamic noise from train pantographs.
\end{abstract}

Keywords:

Noise, railway, pantograph, aerodynamics, prediction, sound, semi-empirical, component-based, high-speed train

\section{Introduction}

At speeds typical of conventional railways the rolling noise is the main source of noise. However, the aerodynamic noise becomes significant at speeds between about 300 and $320 \mathrm{~km} / \mathrm{h}$ and dominant above around $350 \mathrm{~km} / \mathrm{h} \mathrm{[1]}$. Taking into account that high-speed trains exceeding $300 \mathrm{~km} / \mathrm{h}$ are being introduced in several countries, aerodynamic noise becomes an important source of railway noise. Different aerodynamic sources of noise are found in a high-speed train, including the bogies, train nose, inter-coach gaps and pantographs (current collector). Although it is only one of many sources contributing to the overall noise of the train, the pantograph is particularly important as it is located on the train roof and is therefore not so effectively shielded by noise barriers as other sources.

From data acquired using a microphone array during passage of a Train à Grande Vitesse (TGV) Duplex at different train speeds, Mellet et al. [1] found that the pantograph can be identified as a significant noise source at a train speed of $350 \mathrm{~km} / \mathrm{h}$. Aerodynamic noise could be identified with two different mechanisms: vortex shedding noise due to the flow-cylinder interaction generated by the struts that compose the main pantograph body and head and broadband noise from the turbulence generated in the pantograph recess [1].

Kitagawa and Nagakura [2] showed that noise barriers are less efficient in reducing the noise from the pantograph compared with other railway noise sources. They measured the time history of the A-weighted sound pressure level (SPL) when two types of Shinkansen train were passing with speeds of $235 \mathrm{~km} / \mathrm{h}$ and $312 \mathrm{~km} / \mathrm{h}$ over a viaduct with 2

\footnotetext{
${ }^{1}$ Accepted for publication by Journal of Sound and Vibration, 16 January 2017, http://dx.doi.org/10.1016/j.jsv.2017.01.028

*Corresponding author. Tel.:+44 7871658013

Email address: eduardolatorreiglesias@gmail.com (E. Latorre Iglesias)
} 
$\mathrm{m}$ high noise barriers installed on both sides. Prominent peaks in the time-history were seen due to the pantographs, showing that they have a significant contribution to the overall noise radiated by the trains. More examples of acoustic array measurements performed during the passage of different high-speed trains are given in $[3,4,5,6,7]$.

Wind tunnel tests present an alternative method of assessing the aerodynamic noise from train components. Lölgen performed noise tests in the Large-scale Low-noise wind tunnel at Maibara (Japan) using an isolated full-scale pantograph at flow speeds up to $400 \mathrm{~km} / \mathrm{h}$ [8]. He found strong peaks in the noise spectrum due to the vortex shedding from contact strips, horns and stroke-limiting cage. From wind tunnel noise tests on a full-scale pantograph Barsikow and King [5] similarly found the noise from the pantograph head to be significant and governed by distinct peaks due to vortex shedding noise from struts. Lauterbach et al. [9] carried out noise tests in an aeroacoustic wind tunnel using a 1/25 scale ICE3 model and a microphone array. They found the noise generated from the pantograph to be tonal and to dominate the noise spectrum for measured frequencies above $5 \mathrm{kHz}$ ( $200 \mathrm{~Hz}$ at full scale).

Due to the importance of the inflow conditions during wind tunnel tests, Brick et al. [10] used spires upstream of a pantograph mounted on a section of train roof to develop an inflow turbulent boundary layer similar to that present on the train roof. Using a microphone array they concluded that the noise emitted by the pantograph decreased due to the effect of the spires reducing the incident flow speed. Takaishi and Ikeda [11] used a similar approach placing spires upstream of a full-scale pantograph. The spires were optimized to reduce the noise they generated themselves and a noise barrier was placed between the spires and the microphone array.

Anechoic wind tunnel noise tests have also been carried out to assess the effect of noise counter-measures applied to train pantographs. For instance, Sueki et al. [12] assessed the effect of porous materials wrapped around the pantograph components to avoid vortex shedding and to reduce the noise radiation, and Ikeda et al. [13] evaluated the variation in the noise radiation due to modifications of the panhead shape.

Prediction models are gaining importance due to the convenience of addressing the problem at early stages of the design process. Predictions of the aerodynamic noise generation can be made, in principle, by means of Computational Fluid Dynamics (CFD) and Computational AeroAcoustics (CAA) numerical methods. However, in the case of highspeed train components, the large spatial domain and complex geometric configurations required make the current numerical prediction techniques very computationally-intensive.

Computational methods have been applied recently to predict the noise radiated by high-speed pantographs. For example, Sato et al. [14] investigated the noise produced by the knee region of the pantograph. They measured experimentally the noise radiated by different tapered cylinders connected by the knee region and used CFD analysis ( $\kappa-\epsilon$ turbulence model) in order to investigate the flow behaviour around the knee models. Other examples are found in the application by Yu et al. [15] of a hybrid method of Non-Linear Acoustic Solver (NLAS) and Ffowcs Williams-Hawkings (FW-H) acoustic analogy to predict the aerodynamic noise produced by a simplified pantograph type DSA350 for an incident flow speed of $350 \mathrm{~km} / \mathrm{h}$, and in the numerical methods applied by Lei et al. [16] to predict the noise radiated by the pantograph struts, dividing the calculation into an unsteady incompressible flow analysis using the Finite Element Method (FEM) and an acoustic analysis using the Boundary Element Method (BEM).

An option to simplify the numerical simulations is to use a component-based approach, as for example in the physics-based model used by Peers [17] to predict the noise radiated by an aircraft landing gear. In this case the flow field and noise are calculated for isolated components reducing the size and complexity of the geometry. An example of application of this approach for high-speed train pantographs is the numerical study carried out by Liu et al. [18] using Delayed Detached Eddy Simulations (DDES) and FW-H acoustic analogy to predict the noise from a circular cylinder yawed by different angles, approximating the main strut of a train pantograph.

Measurements of noise from cylinders were carried out by Hutcheson and Brooks [19]. Similar tests have been performed by King and Pfizenmaier [20] assessing the effect of the cylinder aspect ratio on the noise radiation for different cylinder cross-sections. Moreau and Doolan [21] assessed experimentally the dependence of the noise on the aspect ratio of a wall-mounted cylinder with a free end. Latorre Iglesias et al. [22] performed anechoic wind tunnel tests studying the dependence of the aerodynamic noise from cylinders on the yaw angle, flow speed, cross-sectional shape, angle of attack and radiation angle (directivity). The results of these experiments can be applied to the study of the aerodynamic noise radiated by a train pantograph.

The use of semi-empirical models based on measured data from individual components can reduce dramatically the computational costs compared with full CFD and CAA simulations. Behr et al. [23] developed a semi-empirical component-based model to assess the noise from a train pantograph based on wind tunnel noise tests with single cylinders. Smith and Chow [24] and Guo [25] developed semi-empirical models to predict the noise from aircraft 
landing gears based on the noise radiated by its different components.

Thompson et al. [26] presented a preliminary adaptation of Guo's model for the aerodynamic noise radiated by different parts of a train such as pantograph, bogie and nose, using the empirical factors from Guo [25]. Latorre Iglesias et al. [27] compared predictions obtained following this approach with available wind tunnel noise tests using a full-size pantograph. It was shown that the model, with modified coefficients, was able to provide fairly good predictions of the overall noise radiated by the pantograph but there were significant differences in the spectral shape. The measured noise spectrum was found to be governed by individual peaks due to the vortex shedding generated by individual struts that are not predicted in the model because the predictions are based on groups of components with a wider spectral shape.

A more detailed review on field and wind tunnel measurements, experimental techniques and numerical and semiempirical models applied to assess and predict the aerodynamic noise from pantographs and another train components is given by Thompson et al. [28].

In the present work a more detailed semi-empirical component-based model is developed for the aerodynamic noise radiated by train pantographs. The noise from each strut is determined independently by approximating it as a cylinder with a particular cross-section and the overall noise from the pantograph is obtained as the incoherent sum of their contributions. Circular, square, elliptical and rectangular sections are considered. The prediction model is based on databases of wind tunnel measurements that are used to determine various empirical constants, which define the shape and amplitude of the noise spectrum generated by each strut. Where acoustic measurements are unavailable the database has been extended using published measurements of related aerodynamic properties. The database is large enough to account for the effect on the vortex shedding noise of the following factors: incident flow speed, diameter, cross-sectional shape, yaw angle, rounded edges, length-to-width ratio, incoming turbulence and directivity. Other factors such as the flow interaction between cylinders, angle of attack, tapered geometry and surface roughness have not yet been included.

The model is validated using previous noise measurements carried out on two different types of pantograph. These are introduced first in Section 2 to show the range of cylinders required in the model. The basic formulation of the proposed model is given in Section 3, the reference cases, for which the initial values of the empirical constants are determined, are described in Section 4 and adjustments made to these constants due to the effect on the vortex shedding noise of the different factors considered in the model are included in Section 5. In Sections 6 and 7 comparisons are given between the predictions obtained by the model and wind tunnel noise measurements of the two pantograph types.

\section{Pantographs studied}

Results from the model developed here will be applied to two typical pantograph types. The first is the DSA350 pantograph shown in Figure 1 with the various components identified. These include circular, elliptical, square and rectangular profiles. The upper and lower arms are inclined by approximately $70^{\circ}$ relative to the flow direction. The cylinder diameters range from $100 \mathrm{~mm}$ for the lower arms to $4 \mathrm{~mm}$ for various small components. This pantograph was tested at flow speeds between 47 and $111 \mathrm{~m} / \mathrm{s}$. The range of Reynolds numbers $\left(R e=D U_{\infty} / \rho\right.$, where $D$ is the width perpendicular to the flow direction, $U_{\infty}$ is the flow speed and $\rho$ is the kinematic viscosity) is therefore $1.3 \times 10^{4}$ to $7.6 \times 10^{5}$. For circular cylinders this covers both the upper subcritical and critical regimes [29].

The second pantograph type considered is an older Japanese type shown in Figure 2. The lower struts are rectangular and the upper ones are circular. The panhead has bars with a rectangular section and a smaller rectangular contact strip mounted on top as shown in the figure. The cylinder diameters range from $26 \mathrm{~mm}$ for the rectangular lower strut to $17 \mathrm{~mm}$ for the horns. Measured results are available in this case for a flow speed of $20 \mathrm{~m} / \mathrm{s}$ although the pantograph would also be used at higher speeds. The range of Reynolds numbers covered is from $1.6 \times 10^{4}$ to $3.4 \times 10^{5}$.

\section{Proposed model}

The following equation is used by various authors $[30,31,32,33]$ to predict the vortex shedding noise radiated by a smooth circular cylinder exposed to a cross-flow. The mean-square pressure at a position $\mathbf{x}$ in the acoustic far-field 


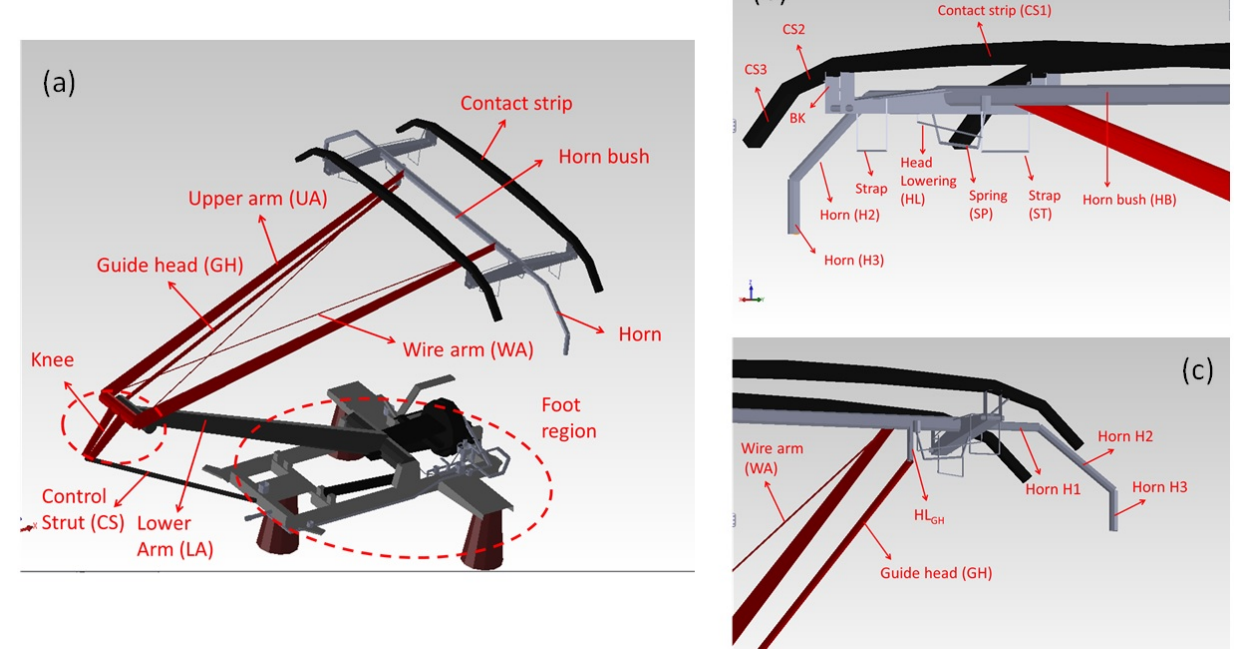

Figure 1: Sketch of the pantograph type DSA350 where the different struts included in the prediction model are detailed. (a) General view. (b) Detail of the pantograph head (I). (c) Detail of the pantograph head (II).

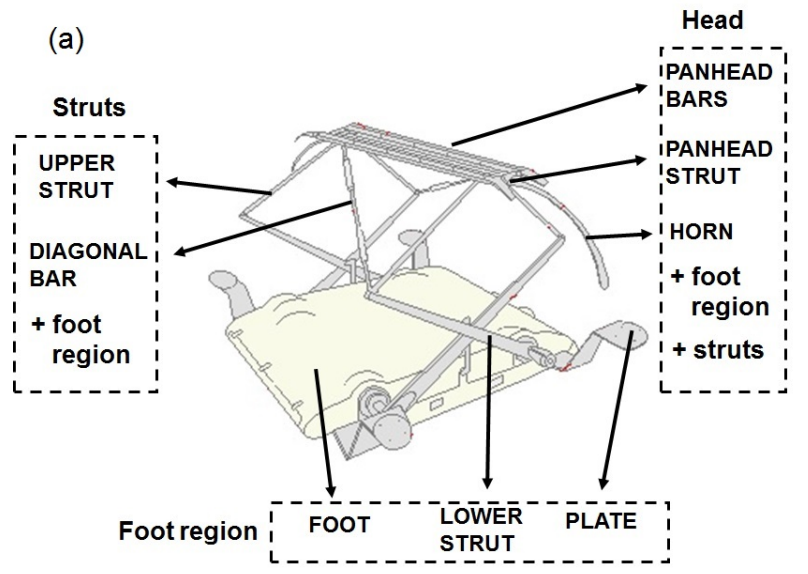

(b)

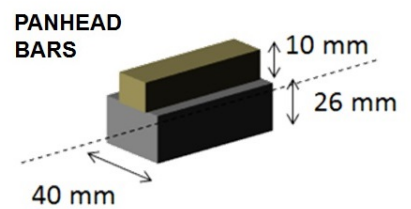

(c)

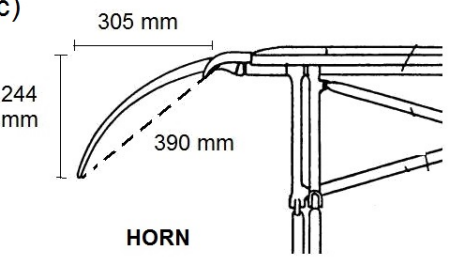

Figure 2: (a) Components of the pantograph included in the prediction model. Figure courtesy of RTRI. (b) Detail of the panhead rectangular bar. (c) Detail of the panhead horn.

is written as

$$
\overline{p^{2}}(\mathbf{x})=\frac{\rho_{0}^{2} U_{\infty}^{6} S t^{2} C_{L \mathrm{rms}}^{2} L l_{c} D}{16 c_{0}^{2} R^{2}} \frac{D_{\mathrm{rad}}(\psi, \phi)}{(1-M \cos (\theta))^{4}}
$$

where $\rho_{0}$ is the air density and $c_{0}$ is the speed of sound. $R$ is the distance between the noise source and the observer, $L$ is the cylinder length and $l_{c}$ is the spanwise correlation length of the surface pressure fluctuations, normalised by the cylinder diameter, $D$. The directivity function $D_{\text {rad }}$ depends on the angle between the axis of the fluctuating lift force (perpendicular to the flow direction) and the receiver position in the azimuthal plane $(\psi)$ and in the elevation plane relative to the azimuthal plane $(\phi)$, as shown in Figure 3. The factor $(1-M \cos (\theta))^{4}$ accounts for the effect of the convective amplification for a dipole source depending on the observer position [34], where $\theta$ is the angle between the direction of the flow and the observer position. The Mach number is defined as $M=U_{\infty} / c_{0}$ and the Strouhal number is defined as

$$
S t=f_{0} D / U_{\infty}
$$


where $f_{0}$ is the vortex shedding frequency. Eq. (1) is based on the assumption that the fluctuating lift force is dominated by the vortex shedding frequency $f_{0} . C_{L r m s}$ is the rms fluctuating lift coefficient. The fluctuating lift coefficient is defined as

$$
C_{L}=\frac{F_{L}}{\frac{1}{2} \rho_{0} U_{\infty}^{2} D L}
$$

where $F_{L}$ is the fluctuating lift.

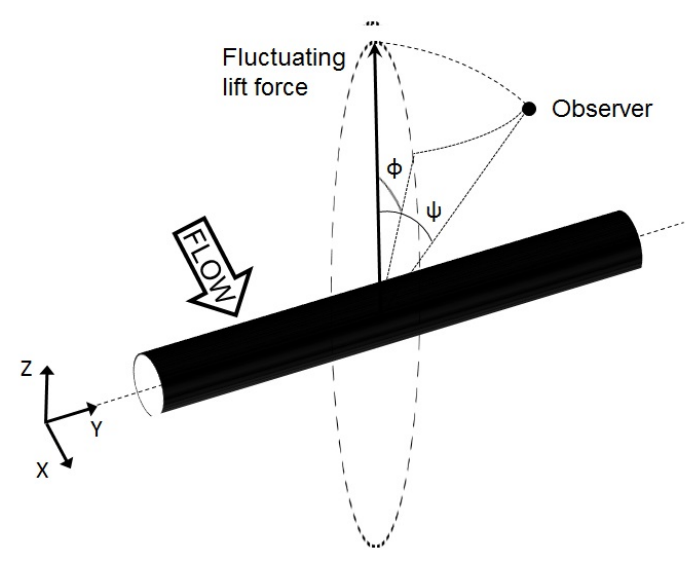

Figure 3: Frame of coordinates where the angles $\phi$ and $\psi$ are defined in relation to the direction of the fluctuating lift force.

\subsection{Amplitude factor}

The corresponding equation from Guo [25], as used by Thompson et al. [26] (neglecting the directivity), is

$$
\overline{p^{2}}(\mathbf{x})=\frac{\left(\rho_{0} c_{0}^{2}\right)^{2} M^{6}}{R^{2}} \int \sum_{i} \eta_{i} S_{i} F_{i}(f) d f
$$

where $\eta$ is the amplitude factor, $S$ is the total surface area of each component of the pantograph, $F(f)$ is a normalized spectrum and the subscript $i$ refers to each strut. It is convenient to express the amplitude factor $\eta$ in terms of the Strouhal number $S t$, the rms fluctuating lift coefficient $C_{L r m s}$ and the correlation length $l_{c}$. If the effects of directivity and convective amplification are ignored and considering only an individual strut, by taking the normalised spectrum $F(f)$ to have an integral over frequency of unity, i.e. $\int F(f) d f=1$, equations (1) and (4) can be equated. Then, the amplitude factor $\eta$ can be obtained as

$$
\eta=\frac{S t^{2} C_{L \mathrm{rms}}^{2} L l_{c} D}{16 S}
$$

This allows empirical databases found in the literature of $S t, C_{L r m s}$ and $l_{c}$ for different cases of cylinders in an airflow to be used to obtain $\eta$, rather than relying only on databases of the noise radiated by cylinders, which are more scarce. Whereas Guo's model is based on groups of components, chosen according to their size, here each component of the pantograph is modelled independently by choosing an appropriate normalised spectrum $F(f)$. A similar approach was followed by Behr et al. [23].

Finally, if the effects of the directivity and convective amplification are included, the mean square sound pressure spectrum radiated by the pantograph can be expressed as the incoherent sum of the spectra of each individual strut as follows:

$$
\overline{p^{2}}(f)=\left(\rho_{0} c_{0}^{2}\right)^{2} M^{6} \sum_{i} \frac{\eta_{i} S_{i} F_{i}(f)}{R_{i}^{2}} \frac{D_{r a d_{i}}(\psi, \phi)}{(1-M \cos (\theta))^{4}}
$$

where $\eta_{i}$ is given by Eq (5), $F_{i}(f)$ is obtained using empirical relations and $D_{\text {rad }}(\psi, \phi)$ is the directivity of the noise radiated by each strut. A speed exponent of 6 is used, which is the theoretical value expected from a dipole source 
such as that produced by the fluctuating lift force. The distance $R$ between the cylinder and the receiver is defined separately for each cylinder.

For each cylinder two normalised spectra are introduced, one to define the vortex shedding peak and the other to define the broadband noise. The subscript $p$ is used for the empirical parameters related to the peak noise and the subscript $b$ is used for the broadband noise. The peak noise spectrum is predicted in narrow frequency steps, in order to have more accuracy in the calculation of the vortex shedding peak frequency and amplitude, and this is then converted to $1 / 3$ octave bands. On the other hand, the broadband noise is calculated directly in $1 / 3$ octave bands.

\subsection{Normalised spectrum}

A "haystack-like" spectral shape is used to model the tonal noise produced by a cylinder undergoing vortex shedding. For the peak noise the model is calibrated using narrow band data so the function used has to provide a narrow spectral shape. It is necessary to control the bandwidth of the peak as this is dependent on various parameters, such as cylinder eccentricity or slenderness and the yaw angle. The following mathematical function, that can provide these features, is chosen:

$$
F_{p}(f)=\frac{a_{p 1}}{\left(\left(f_{0} / f\right)^{2}-\left(f / f_{0}\right)^{2}\right)^{2}+a_{p 2}}
$$

where $f_{0}$ is the vortex shedding frequency. This equation is inspired by the frequency response of a damped single degree of freedom mass-spring system. However, an exponent of 4 is used in the denominator in order to make the slope of the function above and below the peak frequency steep enough that they do not affect the broadband noise which is predicted separately in the model. The value of the parameter $a_{p 1}$ is chosen to force the integral of $F_{p}(f)$ over frequency to be unity.

The relative bandwidth is defined as $B_{f} / f_{0}$, where $B_{f}$ is the bandwidth. This can be chosen by adjusting the parameter $a_{p 2}$. The bandwidth is defined here in terms of points $10 \mathrm{~dB}$ below the peak [22]. Hence, the value of $a_{p 2}$ is given by

$$
a_{p 2}=\frac{1}{2.25}\left(\frac{B_{f}}{f_{0}}\right)^{2}
$$

For the broadband noise spectral shape is useful to control independently the slope of the function at frequencies above and below $f_{0}$. A mathematical function capable of generating a broad "haystack-like" spectral shape is chosen:

$$
F_{b}(f)=\frac{a_{b 1}}{\left(f / f_{0}\right)^{n_{2}}+1+\left(n_{2} / n_{1}\right)\left(f / f_{0}\right)^{-n_{1}}}
$$

This function is arranged so that its maximum occurs at $f_{0}$ which is set equal to the vortex shedding frequency. The exponents $n_{1}$ and $n_{2}$ allow the slope of the function to be controlled at frequencies above and below the vortex shedding frequency. The factor $n_{2} / n_{1}$ is introduced in the denominator to ensure that the maximum of the function occurs at the vortex shedding frequency $f_{0}$ even when $n_{2} \neq n_{1}$. Finally a constant $a_{b 1}$ is included, its value being chosen to ensure that the integral of $F_{b}(f)$ over frequency is equal to unity, as explained above for $a_{p 1}$. The amplitudes of the two components are determined by the respective factors $\eta_{i}$.

\section{Reference cases}

The values of the amplitude factor, speed exponent and constants of the normalised spectra are first obtained for two reference cases. These are a circular and a square cylinder in clean flow (no turbulence), with zero yaw angle and an angle of attack of $0^{\circ}$. It is assumed that the cylinders are long enough that end effects can be neglected. For the square cylinder sharp edges are assumed. Reynolds numbers in the upper subcritical flow regime $\left(1.0 \times 10^{4}<R e<\right.$ $1.4 \times 10^{5}$ ) are considered. Subsequently, the influence of various factors (flow speed and flow regime, yaw angle, crosssection shape, aspect ratio, angle of attack, rounded edges and incoming turbulence) will be addressed by means of corrections applied to the values of the empirical constants obtained for these initial cases, using experimental results from various studies. 


\subsection{Peak noise}

For the peak noise, the amplitude factor is obtained by means of Eq. (5) using experimental data found in the literature from measurements of Strouhal number, rms fluctuating lift coefficient and correlation length. The empirical constants of the normalised spectrum and the speed exponent are also determined using the experimental data presented in [22]. Table 1 shows the parameters used for the two reference cases to obtain the amplitude factor of the peak noise $\eta_{p}$.

Table 1: Values of Strouhal number $S t$, rms fluctuating lift coefficient $C_{L \mathrm{rms}}$ and normalised correlation length $l_{c}$ found in the literature and used to obtain the amplitude factor of the peak noise, $\eta_{p}$, for circular and square cylinders.

\begin{tabular}{|c|c|c|c|}
\hline & $S t$ & $C_{L \mathrm{rms}}$ & $l_{c}$ \\
\hline Circular & 0.190 (from [22]) & 0.5 (from [35]) & 3.0 (from [35]) \\
\hline Square & 0.125 (from [22]) & 1.0 (from [36]) & 5.6 (from [37]) \\
\hline
\end{tabular}

For circular cylinders the values of $C_{L \mathrm{rms}}$ and $l_{c}$ vary slightly in the upper subcritical range $\left(1.0 \times 10^{4}<R e<\right.$ $1.4 \times 10^{5}$ ) but they have opposite trends so their effects tend to cancel each other [35, 38, 39]. Considering the smallest component of the pantograph and the lowest flow speed $(D=4 \mathrm{~mm}$ and $U=32 \mathrm{~m} / \mathrm{s}$ for the pantograph type DSA350 and $D=12 \mathrm{~mm}$ and $U=20 \mathrm{~m} / \mathrm{s}$ for the Japanese pantograph), the lowest value of Reynolds number considered will be above $1.0 \times 10^{4}$. For square cylinders, in the Reynolds number range considered for the reference case, the flow separates at the front edges of the cylinder and is not reattached to its surface [36, 40] so Reynolds number effects can be neglected. The method used to measure the fluctuating lift coefficient and the correlation length can introduce variations in the values obtained, as shown by Norberg [41] and Duarte Ribeiro [42], introducing an additional uncertainty in the prediction.

Table 2 shows the values of the empirical constants obtained for the peak noise of circular and square cylinders. The factor $a_{p 2}$ is selected to ensure that the bandwidth of the normalised spectrum matches the experimental data in [22]. These noise measurements were carried out in an open-jet anechoic wind tunnel using cylinders with different cross-sections. The average value of the relative bandwidth for five flow speeds was $B_{f} / f_{0}=0.16$ for the circular case and $B_{f} / f_{0}=0.28$ for the square case. The value of the speed exponent $\alpha$ is also based on the results from [22].

Table 2: Values of the empirical constants used for the peak noise of the circular and square cylinders for use in narrow-band spectra.

\begin{tabular}{|c|c|c|c|c|}
\hline & $\eta_{p}$ & $a_{p 2}$ & $\alpha$ & $S t_{0}$ \\
\hline Circular & $5.37 \times 10^{-4}$ & 0.011 & 6.0 & 0.190 \\
\hline Square & $1.40 \times 10^{-3}$ & 0.035 & 6.0 & 0.125 \\
\hline
\end{tabular}

\subsection{Broadband noise}

A different approach is followed in the case of the broadband noise spectrum. The values of $n_{1}$ and $n_{2}$, which control the width of the peak, are chosen to fit the experimental results from [43]. As shown in [43] the spectral shape of the broadband noise is nearly independent of the cylinder cross-section and flow speed in the Reynolds number range considered here.

To predict the dependence of the amplitude factor, $\eta_{b}$, on the different factors, it is necessary to understand the physical mechanisms involved in the noise generation. To simplify the problem it is assumed that the variation of $\eta_{b}$ due to, for example the yaw angle, is related to the corresponding variation of the amplitude factor for the peak noise, $\eta_{p}$. The ratio $\eta_{p} / \eta_{b}$ is calculated from experimental results presented in [22, 43].

Figure 4 (a) shows the difference between the overall sound pressure level (OASPL) of broadband and peak noise for five flow speeds and different cylinder cross-sections at a yaw angle of $0^{\circ}$. The variation of the results with the type of cylinder cross-section is small. An average value for all the cylinder cross-sections of $-12.4 \mathrm{~dB}$ is obtained. This is represented in the prediction model by the values of $\eta$. Considering that the level difference is $10 \log _{10}\left(\eta_{b} / \eta_{p}\right)$, then $\eta_{b}=0.057 \eta_{p}$. This is used as the reference relation between the peak and broadband noise amplitude. 


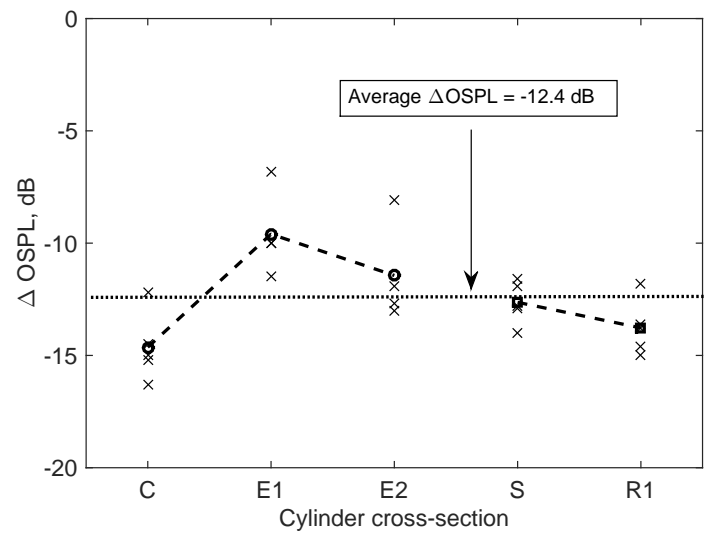

Figure 4: Averaged difference between broadband and peak noise overall levels for the five flow speeds vs. cylinder cross-section [43]. C stands for circular cylinder, E1 for elliptical cylinder with eccentricity 0.63 , E2 for elliptical cylinder with eccentricity 0.75 , S for square cylinder and R1 for rectangular cylinder with slenderness ratio 1.33 .

Table 3 shows the values of the empirical constants used for the broadband noise of the circular and square cylinders. The values of $\alpha$ are those obtained in [43]. These are different from 6, as used for the peak noise, especially for the circular cylinder. Due to the different speed exponent between the peak and broadband noise the relation $\eta_{b}=0.057 \eta_{p}$ only applies for $R e=2.59 \times 10^{4}$ for the circular cylinder and $R e=3.45 \times 10^{4}$ for the square cylinder, which correspond to a flow speed of $31.5 \mathrm{~m} / \mathrm{s}$ during the experiments in [43].

Table 3: Values of the empirical constant used for the broadband noise of the circular and square cylinder in $1 / 3$ octave bands.

\begin{tabular}{|c|c|c|c|c|c|c|}
\hline & $\eta_{b}$ & $n_{1}$ & $n_{2}$ & $n_{2} / n_{1}$ & $\alpha$ & $S t_{0}$ \\
\hline Circular & $8.50 \times 10^{-7}$ & 1.35 & 1.35 & 1.00 & 7.0 & 0.190 \\
\hline Square & $4.8 \times 10^{-5}$ & 2.50 & 1.30 & 0.52 & 6.1 & 0.125 \\
\hline
\end{tabular}

\subsection{Results}

Figure 5 compares the experimental results and predictions for a circular cylinder normal to the flow direction. The values of the empirical constants used are as given in Tables 2 and 3. To obtain the overall noise, the peak noise is calculated first as a narrow band spectrum and then converted into 1/3 octaves. Then, the peak noise and the broadband noise are added incoherently. Good agreement is found between the predictions and measurements, with a difference in the OASPL of $-0.9 \mathrm{~dB}$ for the circular cylinder and $2.9 \mathrm{~dB}$ for the square cylinder.

Figure 5(a) shows that the spectral shape is predicted well at the peak but in the measurements there is a secondary peak between 250 and $400 \mathrm{~Hz}$ which broadens the peak when the results are converted to 1/3 octaves. This "hump" was observed in [22] for the circular cylinder for all the flow speeds but it was not found for other cylinder crosssections. Moreau and Doolan [21] found that for a circular cylinder at certain aspect ratios a second peak appeared at a frequency around $10 \%$ lower than the main vortex shedding peak and with smaller amplitude. The same phenomenon was found by King and Pfizenmaier [20] using circular and rectangular cylinders for certain cylinder aspect ratios. Here the secondary peak is around $25 \%$ lower in frequency than the main peak. Nevertheless, its presence does not affect the overall SPL so it has not been taken into account in the model.

\subsection{Directivity}

The directivity of the peak noise radiated by cylinders can be approximated by a dipole directivity [22]

$$
D_{\text {rad }}(\psi, \phi)=\cos (\psi) \cos (\phi)
$$



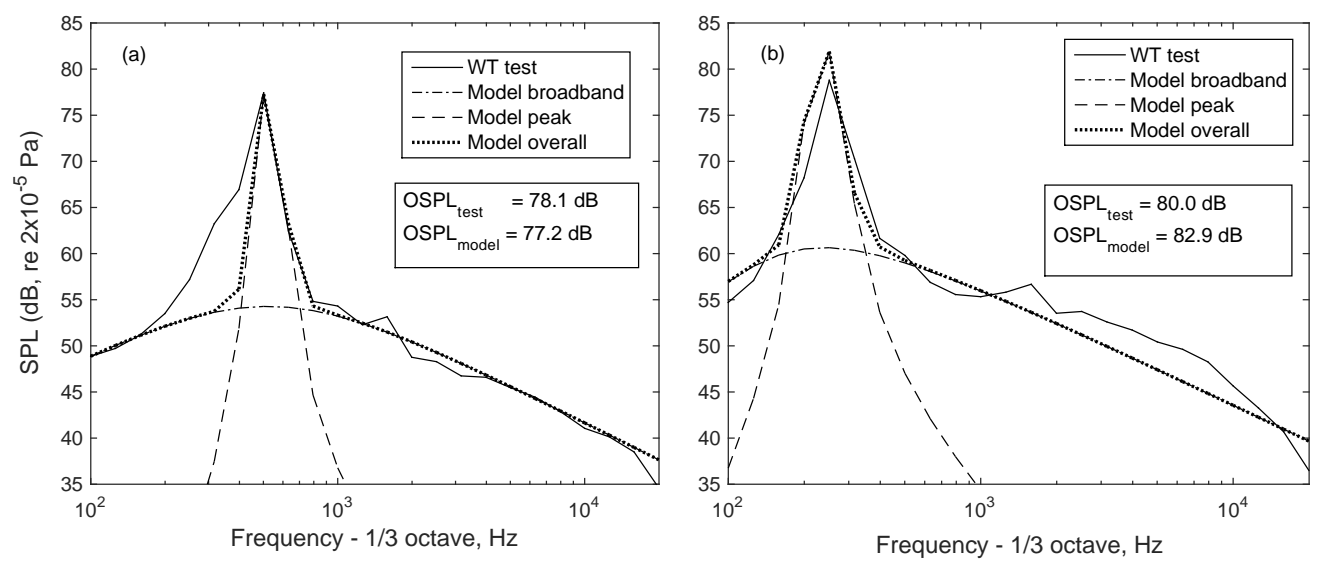

Figure 5: Comparison between the predicted and measured broadband noise spectrum, for a flow speed of $31.5 \mathrm{~m} / \mathrm{s}$ and a yaw angle $0^{\circ}$. Experimental data taken from [22]. Corrections due to the cylinder aspect ratio were applied as shown in Section 5.1 below. (a) Circular cylinder (b) Square cylinder.

where $\phi$ and $\psi$ are the azimuthal and elevation angles with respect to the direction of the fluctuating lift force acting on the cylinder (see Figure 3), which is equivalent to the axis of the dipole source. The symmetry in the radiation in the vertical planes both parallel and perpendicular to the flow direction was shown in [27], together with the error introduced by this assumption. The broadband noise can be considered as omnidirectional, that is $D_{\mathrm{rad}}(\psi, \phi)=1$, the error introduced by this simplification being less than $+/-5 \mathrm{~dB}$ [43]. The directivity of the peak and broadband noise was found to be independent of the flow speed and yaw angle.

\section{Model adjustments}

Having established the two reference cases, correction terms are introduced in this section to allow for the effects of various parameter changes.

\subsection{Effect of the aspect ratio}

For sufficiently long cylinders the end effects can be neglected. This implies that, if the length of the cylinder is increased, the Strouhal number will remain constant and the SPL will increase according to $10 \log _{10}(L)$. However, for smaller aspect ratios $L / D$ the dependence of the Strouhal number and SPL on the cylinder length will differ from this.

Experimental data obtained by King and Pfizenmaier [20] and Moreau and Doolan [21] are used to assess the effect of the aspect ratio on the vortex shedding noise from a circular cylinder. In the prediction model it is assumed that the vortex shedding is triggered for aspect ratios larger than 4 and that an aspect ratio $L / D=25$ is sufficient to neglect any end effects [20]. In the prediction model, due to a lack of data, the corrections due to the aspect ratio obtained for circular cylinders are also applied to elliptical cylinders while the corrections for square cylinders are also applied to rectangular cylinders.

Figure 6(a) shows the effect of the aspect ratio on the Strouhal number for a circular cylinder. The results are shown relative to that for an aspect ratio 25 , denoted $S t_{0}$. These results were fitted with a linear function of $\log _{10}(L / D)$ using a least squares method.

$$
S t_{i} / S t_{0}=0.36 \log _{10}(L / D)+0.50, \text { for } \quad 4 \leq L / D<25
$$

Figure 6(b) shows the dependence of the peak noise SPL radiated by a circular cylinder on the aspect ratio. The results are shown as the difference between the SPL for a particular aspect ratio and that for an aspect ratio of 25 . The effect of the decrease of the cylinder length was first corrected by adding a factor $10 \log _{10}(25 D / L)$ to the measured SPL. For $L / D>10.5$ the results were fitted by a least squares method using a linear function. For $L / D \leq 10.5$ a constant value is used, obtained as the average of all the results. In this case the noise does not decrease when the 

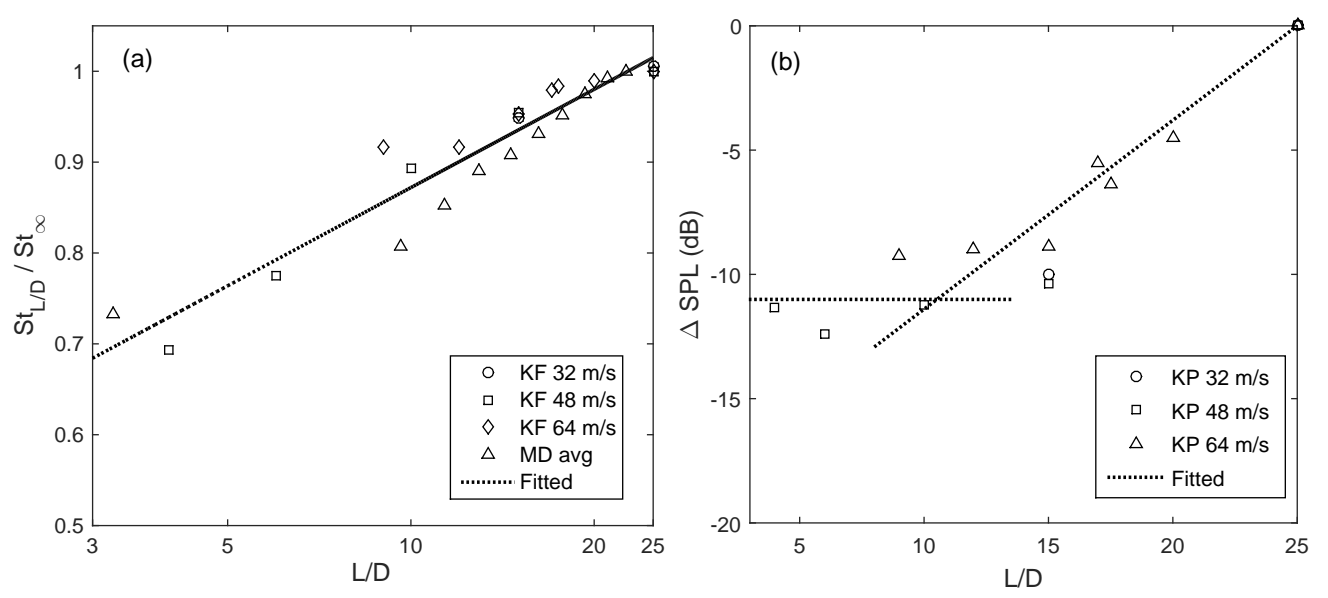

Figure 6: Variation of St and SPL with the cylinder aspect ratio measured by King and Pfizenmaier (KP) [20] and Moreau and Doolan (MD) [21] for a circular cylinder. (a) Ratio between the Strouhal number obtained for different aspect ratios and for $L / D=25$. (b) Difference between the SPL measured for different aspect ratios and $L / D=25$.

cylinder is shorter (after applying cylinder length correction) probably due to the influence of the broadband noise. From the results shown in Figure 6(b)

$$
10 \log _{10}\left(\eta_{i} / \eta_{0}\right)=0.85(L / D)-21.17, \text { for } L / D>10.5
$$

where $\eta_{i} / \eta_{0}$ is the ratio between the value of the amplitude factor of the peak noise with and without the aspect ratio correction. Figure 6(b) shows variations less than $+/-2 \mathrm{~dB}$ between the measured and fitted results.

For square cylinders Figure 7(a) shows the ratio between the Strouhal number obtained with different aspect ratios and with an aspect ratio 25. The decrease of the Strouhal number with the reduction of the aspect ratio is smaller than for circular cylinders. A linear relation is obtained after applying least squares fitting to the results. There is a discontinuity between the results obtained by Moreau and Doolan for $L / D=13.6$ and by King and Pfizenmaier [20] for $L / D=16.7$. The results from Moreau and Doolan for $8 \leq L / D \leq 13.6$ have been neglected for the curve fitting (they found values of $S t$ up to 0.13 compared with 0.125 in Table 2 for large aspect ratio). The effect of aspect ratio is included in the prediction model for square cylinders as follows

$$
S t_{i} / S t_{0}=0.127 \log _{10}(L / D)+0.823, \text { for } 2 \leq L / D<25
$$

Figure 7(b) shows the difference in SPL due to changes in $L / D$. Experimental results $[21,20]$ are again shown which were corrected in the graph by a factor $10 \log _{10}(25 D / L)$ to account for the variation of the SPL with cylinder length. For $2 \leq L / D \leq 6$ the SPL remains nearly constant at around $-15 \mathrm{~dB}$ whereas for $6<L / D \leq 25$ the dependency of the SPL on the aspect ratio corresponds to a quadratic function. The results for $2 \leq L / D \leq 6$ are probably more affected by the broadband noise. These results, and those for the circular cylinders, suggest that the broadband noise does not change significantly with the cylinder aspect ratio so in the prediction model it is assumed that the broadband noise is independent of it.

The variation of the amplitude of the peak noise with the aspect ratio of a square cylinder is included in the model as follows:

$$
10 \log _{10}\left(\eta_{i} / \eta_{0}\right)= \begin{cases}-16.0, & \text { if } 2 \leq L / D \leq 6 \\ 5.62 \times 10^{-2}(L / D)^{2}-2.58(L / D)-29.45, & \text { if } 6<L / D<25\end{cases}
$$

The differences between measured and fitted results are between $+/-3 \mathrm{~dB}$, as shown in Figure 7(b).

\subsection{Dependence on the Reynolds number for circular struts}

The behaviour of the air flow around a circular cylinder strongly depends on the Reynolds number. In the Reynolds number range considered here for the pantograph, it is assumed that the results for square cylinders and rectangular 

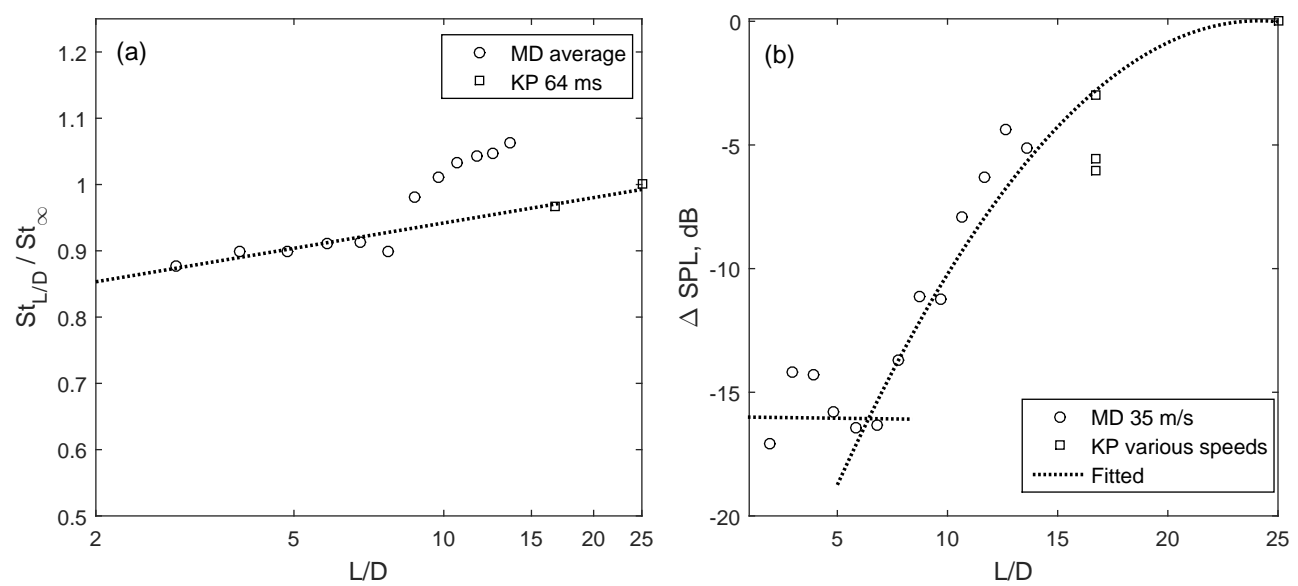

Figure 7: Variation of St and SPL with the cylinder aspect ratio measured by Moreau and Doolan (MD) [21] for a flow speed of $35 \mathrm{~m} / \mathrm{s}$ and averaged results for flow speeds of 25, 30 and $35 \mathrm{~m} / \mathrm{s}$ and by King and Pfizenmaier (KP) [20] for various flow speeds, for a square cylinder. (a) Ratio between the Strouhal number obtained for different aspect ratios $L / D$ and for $L / D=25$. (b) Difference between the SPL measured for different aspect ratios $L / D$ and for $L / D=25$

cylinders with moderate slenderness ratios (i.e. $0.5 \leq B / D \leq 2.0$ ) can be treated as independent of the Reynolds number, as the flow separates at the front edges and is not reattached to the cylinder surface [36, 40, 44]. The flow behaviour around elliptical cylinders is also dependent on the Reynolds number but due to a lack of data only the circular cylinders are considered in this section. The pantographs contain circular struts with flow in both the subcritical and critical flow regimes. These two regimes are considered separately. For the subcritical regime the variations of the spectral amplitudes of the peak and broadband noise are based on the speed dependence obtained by Latorre Iglesias et al. [22]. For the critical regime the corrections in the noise spectrum amplitude and shape are based on the experimental results obtained by Fujita [45].

The first step is to determine the critical Reynolds number $R e_{c}$, that for which the transition between the subcritical and critical regimes occurs. For a circular cylinder exposed to a normally incident clean flow the transition between the subcritical and critical flow regimes occurs for Reynolds numbers between $1.0 \times 10^{5}$ and $2.0 \times 10^{5}$, at which a sudden increase of the Strouhal number and a decrease of the fluctuating lift coefficient occur [38, 46]. In the prediction model, $\operatorname{Re}_{c}=1.4 \times 10^{5}$ is used for a circular cylinder perpendicular to the flow with no turbulence.

For inclined cylinders and/or turbulent flow the value of $R e_{c}$ may change. Measuring the pressure distribution about a circular cylinder, Bursnall et al. [47] found that the critical Reynolds number $R e_{c}$ based on the freestream flow speed $U_{\infty}$ is independent of the cylinder yaw angle (for yaw angles up to $60^{\circ}$ ). These results have been confirmed in recent numerical studies carried out by Liu et al. [18]. In the prediction model, in order to define whether the flow around a circular cylinder is in the subcritical or critical regime, the Reynolds number is therefore calculated using the free stream flow speed $U_{\infty}$, even for those cases when the cylinder is yawed. However, the incoming turbulence affects the value of $R e_{c}$, as explained in more detail in Section 5.7. This has to be considered in advance before determining whether the flow conditions around the cylinder are those for the subcritical or critical regimes.

\subsubsection{Subcritical flow regime}

In the subcritical regime the dependence of the noise amplitude on the flow speed is given by the speed exponent $\alpha$, values of which are given in Table 2 and Table 3 for the peak and broadband noise. Figure 8 shows the difference between the OASPL predicted using the model and measured results $[22,20]$ for circular and square cylinders arranged perpendicular to the flow direction, for different Reynolds numbers. The differences in OASPL are less than $3 \mathrm{~dB}$ except for the circular cylinder at the highest Reynolds numbers measured by King and Pfizenmaier, for which the difference increases to $5 \mathrm{~dB}$. They found a decrease of the speed exponent $\alpha$ for flow speeds above $50 \mathrm{~m} / \mathrm{s}(R e \approx$ $7.0 \times 10^{4}$ ) which leads to this discrepancy. 


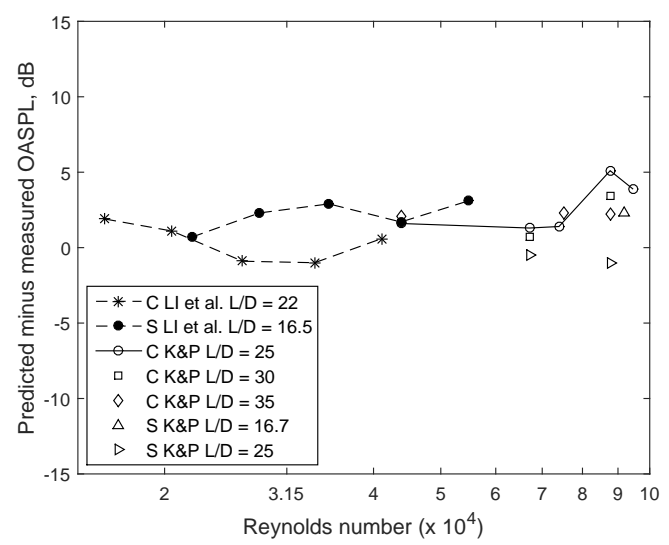

Figure 8: Differences between the predicted OASPL and that measured by Latorre Iglesias (LI) et al. [22] and by King and Pfizenmaier (KP) [20] for circular and square cylinders normal to the incident flow, for different Reynolds numbers and aspect ratios. Aspect ratio corrections (see Section 5.1) were applied to the predictions for $L / D<25$.

\subsubsection{Critical flow regime}

Figure 9 shows the variation of the Strouhal number $S t$ of circular cylinders with the Reynolds number $R e$ in the high subcritical, critical and supercritical flow regimes. Two different sets of results are compared: those obtained by Schewe from measurements of the fluctuating lift coefficient [38] and those obtained by Fujita from measurements of vortex shedding noise [45]. Fujita found fluctuations of the Strouhal number for some Reynolds numbers [45]. In these cases the results shown in Figure 9(a) are the averaged values. The trend followed by the two sets of results are in good agreement, except for $1.0 \times 10^{6} \leq R e<2.0 \times 10^{6}$ but in this range the results from Schewe show duplicated values of the Strouhal number.

From the results shown in Figure 9(a) the dependence of the Strouhal number on the Reynolds number in the critical flow regime is included in the prediction model as follows

$$
S t_{i} / S t_{0}= \begin{cases}1, & \text { if } R e \leq R e_{c} \\ 2.2, & \text { if } R e_{c}<R e \leq 1 \times 10^{6} \\ 1, & \text { if } 1 \times 10^{6}<\operatorname{Re} \leq 3.2 \times 10^{6} \\ 1.13 \log _{10}(\operatorname{Re})-6.38, & \text { if } \operatorname{Re}>3.2 \times 10^{6}\end{cases}
$$

Figure 9(b) shows the difference between the SPL at different Reynolds numbers and at $\operatorname{e}_{c}$ from Fujita [45]. These results are compared with the expected variation of the SPL due to changes in the value of $\eta$, derived from equation (5), using the Strouhal number, rms fluctuating lift coefficient and correlation length measured by Schewe [38] and Schlinker [39]. All data are normalised such that $\Delta \mathrm{SPL}=0 \mathrm{~dB}$ is obtained at $\operatorname{Re}_{c}$. However the lowest Reynolds number measured by Fujita was $R e=2.5 \times 10^{5}$. The value $\Delta \mathrm{SPL}=-5.3 \mathrm{~dB}$ at $R e=2.5 \times 10^{5}$ was therefore chosen to correspond to Schewe's results.

The results in Figure 9(b) show that a similar trend is followed by the various results for Reynolds numbers up to $3.5 \times 10^{5}$, with a significant decrease of the SPL. For Reynolds numbers higher than $3.5 \times 10^{5}$, however, the SPL predicted from $S t, C_{L \mathrm{rms}}$ and $l_{c D}$ remains fairly constant (variations less than $+/-5 \mathrm{~dB}$ ) while the SPL measured by Fujita has a steep increase for Reynolds numbers greater than $4.9 \times 10^{5}$. For Reynolds numbers above the critical Reynolds number the vortex shedding process becomes more disorganised and no regular vortex shedding can be observed [48] hence the abrupt decrease of $\eta$. For Reynolds numbers above around $1.0 \times 10^{6}$ narrow band lift fluctuations appear [38] that are probably related with the slight increase of $\eta$. However, in this range of Reynolds number the noise increases proportionally to $U^{7.3}$, which is a speed dependence significantly higher than that for a dipole source.

To account for this in the prediction model it is assumed that, for Reynolds numbers higher than $R e_{c}$, the variation 

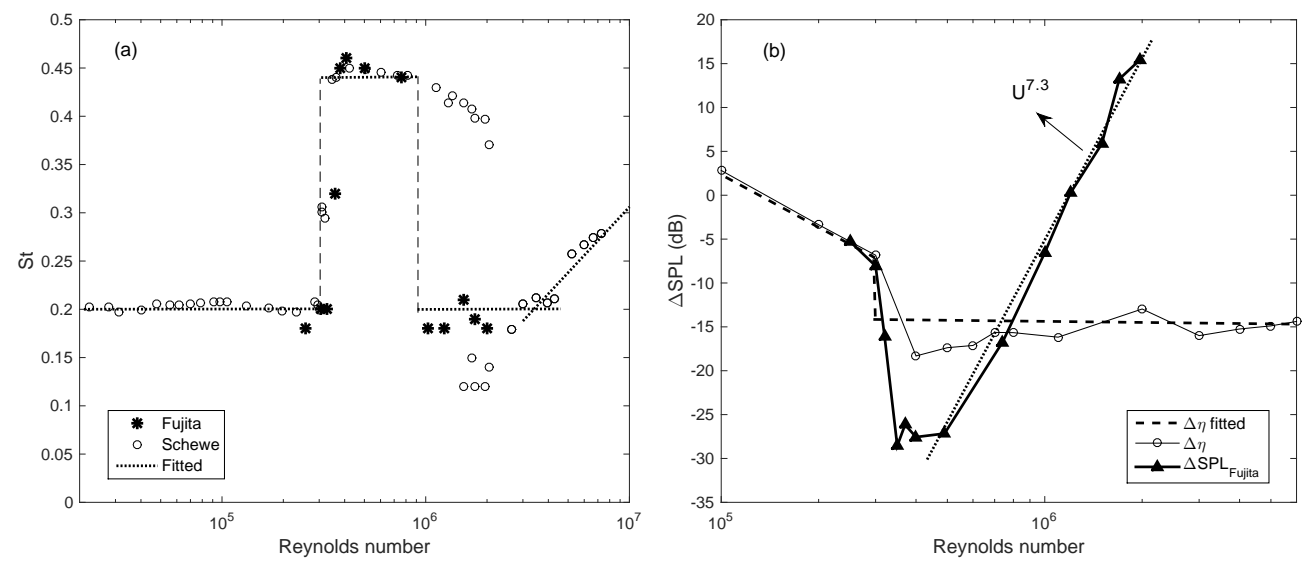

Figure 9: (a) Variation of the Strouhal number $S t$ with the Reynolds number Re obtained by Schewe measuring fluctuating lift [38] and by Fujita measuring vortex shedding noise [45]. (b) Difference between the peak SPL for $R e=1.4 \times 10^{5}$ (beginning of the transition to critical regime) and the peak SPL for higher Reynolds numbers. The results measured by Fujita [45] are compared with the expected decrease of the SPL due to the variation of $\eta$, according to the values of Strouhal number $S t$, fluctuating lift coefficient $C_{L r m s}$ and normalized correlation length $l_{c D}$ measured by Schewe [38] and Schlinker [39]

of the amplitude factor is given by

$$
\eta_{i} / \eta_{0}= \begin{cases}-24.17 \log _{10}(R e)+124.4, & \text { if } R e_{c} \leq R e \leq 2 R e_{c} \\ 0.032, & \text { if } R e>2 R e_{c}\end{cases}
$$

as shown by the dotted line in Figure 9(b). The results in Figure 9(b) shows that, for Reynolds numbers below $5 \times 10^{5}$, the SPL and $\eta_{i} / \eta_{0}$ follow a similar trend so it is assumed that the noise only depends on the empirical factor $\eta$. In order to avoid the noise increasing with the flow speed the equation (6) is multiplied by a factor $R e_{c}^{6} / R e^{6}$. For Reynolds numbers above $7 \times 10^{5}$ this factor is removed and a speed exponent $\alpha=7.3$ is assumed both for the peak and broadband noise in order to obtain the noise dependence on the Reynolds number as shown in Figure 9(b). Hence the increase of the SPL with the Reynolds number is given by

$$
\Delta S P L= \begin{cases}10 \log _{10}\left(\eta_{i} / \eta_{0}\right), & \text { if } R e_{c} \leq \operatorname{Re} \leq 7 \times 10^{5} \\ 10 \log _{10}\left(\left(\operatorname{Re} / 7 \times 10^{5}\right)^{7.3}\right), & \text { if } \operatorname{Re}>7 \times 10^{5}\end{cases}
$$

\subsection{Effect of the cross-section shape}

To consider the effect of cross-section shape, circular and square cylinders are used as reference cases in the prediction model, as explained in Section 4. The circular cylinder is a particular case of an elliptical cylinder with eccentricity $e=0$, while the square cylinder is a particular case of a rectangular cylinder with slenderness ratio $B / D=1$. The eccentricity is defined as $e=\sqrt{\left(1-(D / B)^{2}\right)}$ where $D$ is the dimension perpendicular to the flow and $B$ is the streamwise dimension. Changes in the eccentricity of elliptical cylinders and slenderness of rectangular cylinders will lead to variations of the amplitude and shape of the noise spectrum. In this section the correction factors applied to the reference cases to account for these effects are introduced.

\subsubsection{Dependence on the eccentricity of elliptical cylinders}

For elliptical cylinders Figure 10 shows the dependence of the Strouhal number and the SPL on the eccentricity, the results being normalised by those for a circular cylinder as measured by King and Pfizenmaier [20] for the Strouhal number and as measured by Latorre Iglesias et al. [22] for the noise amplitude. Results are included in Figure 10 from various authors. For the results from Modi and Wiland [49] the change in noise level is calculated from $10 \log _{10}(\eta)$, in 

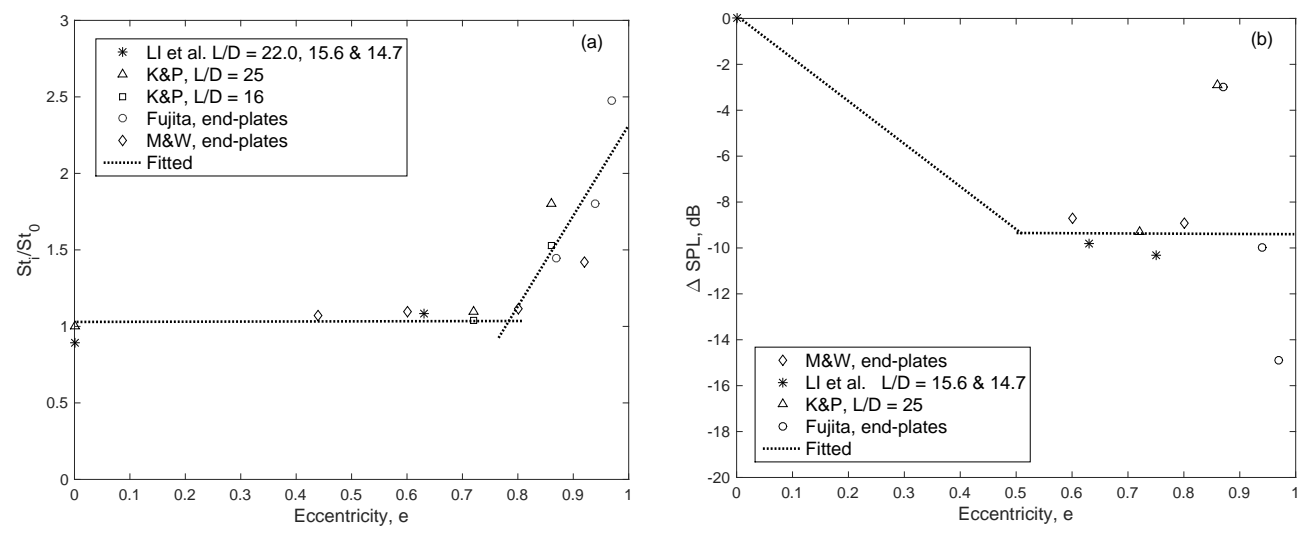

Figure 10: Influence of the eccentricity $e$ of elliptical cylinders on St and SPL. Data from Latorre Iglesias (LI) et al. [22], Modi and Wiland (M\&W) [49], King and Pfizenmaier (K\&P) [20] and Fujita [50]. (a) Variation of the Strouhal number St with the cylinder eccentricity $e$. (b) Variation of the ratio between the noise radiated by an elliptical cylinder and a circular cylinder with the cylinder eccentricity $e$.

which the value of $\eta$ is derived from equation (5) using the measured values of Strouhal number, rms fluctuating lift coefficient and correlation length.

For elliptical cylinders the dependency of the Strouhal number on the cylinder eccentricity $e$ implemented in the prediction model is based on the fitted data shown in Figure 10(a). The proposed relation is

$$
S t_{i} / S t_{0}= \begin{cases}1, & \text { if } 0 \leq e \leq 0.78 \\ 5.9 e-3.6, & \text { if } e>0.78\end{cases}
$$

The variation of the amplitude of the peak noise with the cylinder eccentricity is shown in Figure 10(b). The dependence of the amplitude factor on the cylinder eccentricity can be obtained from these results by means of the expression $\Delta \mathrm{SPL}=10 \log _{10}\left(\eta_{i} / \eta_{0}\right)$. No data was found for eccentricities lower than 0.6. In order to avoid a discontinuity in the value of the amplitude factor a linear decay is supposed for eccentricities between 0 and 0.5 . The following relation is used in the model

$$
10 \log _{10}\left(\eta_{i} / \eta_{0}\right)= \begin{cases}-18.8 e, & \text { if } e<0.5 \\ -9.4, & \text { if } 0.5 \leq e \leq 1.0\end{cases}
$$

According to the results obtained by Latorre Iglesias et al. [22] the speed exponent $\alpha$ can be assumed to be constant for different cylinder eccentricities, even though a large variability was obtained at different yaw angles [22]. However, the relative bandwidth measured for elliptical cylinders was found to be larger than that for a circular cylinder. The relative bandwidth was only measured for cylinder eccentricities 0.63 and 0.75 and no other additional information was found in the literature. The relative bandwidth was found to increase by a factor of around 7 compared with that for a circular cylinder for eccentricities of both 0.63 and 0.75 . A linear increase of the relative bandwidth is assumed for eccentricities lower than 0.6 and a constant value is assumed for larger eccentricities, the validity of this assumption not being confirmed.

$$
B_{f_{i}} / B_{f_{0}}= \begin{cases}12.4 e+1, & \text { if } e<0.6 \\ 7.1, & \text { if } e \geq 0.6\end{cases}
$$

The value of 7.1 used in equation (20) is based on the average value of the relative bandwidth, $B_{f_{i}} / f_{0}$, obtained in [22] for elliptical cylinders with eccentricities 0.63 and 0.75 with respect to that for a circular cylinder.

\subsubsection{Dependence on the slenderness of rectangular cylinders}

For rectangular cylinders Figure 11 shows the dependence of the Strouhal number and the amplitude of the peak noise on the slenderness ratio, the results being normalised by the values for a square cylinder. The data were obtained from the results measured by King and Pfizenmaier [20], Knisely [36] and Latorre Iglesias et al. [22]. 

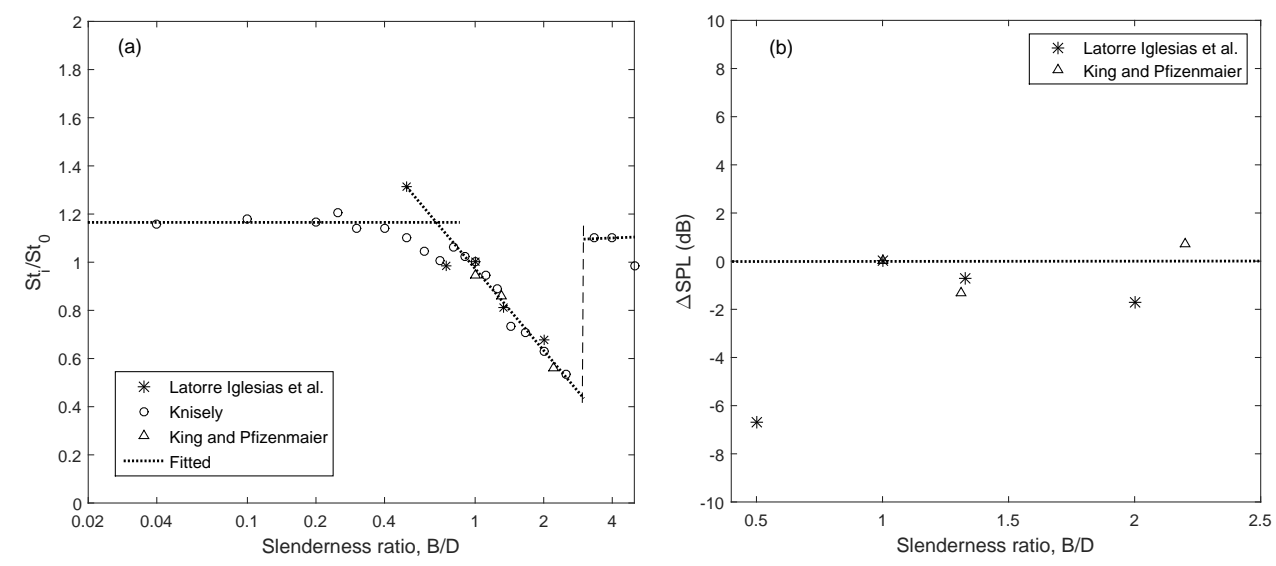

Figure 11: Effect of the slenderness ratio $(B / D)$ of a rectangular cylinder on the vortex shedding noise. Results include those from King and Pfizenmaier [20], Knisely [36] and Latorre iglesias et al. [22]. (a) Variation of the Strouhal number with the slenderness ratio normalised by the Strouhal number for a square cylinder $(B / D=1)$. (b) Variation of the noise with the slenderness ratio of rectangular cylinders with respect to the noise for a square cylinder $(B / D=1)$.

The Strouhal number for a rectangular cylinder with slenderness $B / D$ is defined from the results shown in Figure 11(a) as follows

$$
S t_{i} / S t_{0}= \begin{cases}1.15, & \text { if } B / D \leq 0.7 \\ -1.13 \log _{10}(B / D)+0.97, & \text { if } 0.7 \leq B / D \leq 3 \\ 1.10, & \text { if } B / D>3\end{cases}
$$

where $S t_{0}$ is the Strouhal number for a square cylinder.

The noise level differences shown in Figure 11(b) are relatively small (less than $+/-2 \mathrm{~dB}$ ) apart from at a slenderness ratio of 0.5 where the difference is around $-6.5 \mathrm{~dB}$. It is therefore assumed that the value of the amplitude factor for the peak noise is independent of the cylinder slenderness. There is a significant difference between the amplitude factor for a slenderness of 0.5 and the other results, this being probably caused by the small cylinder aspect ratio $(L / D=7.5)$ [22]. Even though a correction due to the different aspect ratios among the cylinders used to obtain the results shown in Figure 11(b) has been applied in the prediction model according to equation (14). For large slenderness ratios $(B / D>>2.0)$ the flow conditions around the cylinder may be different and the flow may reattach on the cylinder surface, leading to changes in the noise radiation. This has not been considered in the prediction model.

The relative bandwidth is assumed to be independent of the cylinder slenderness, based on the results measured by Latorre Iglesias et al [22] using a square cylinder and rectangular cylinders with slenderness ratio 1.33 and 2 .

Latorre Iglesias et al. [22] found the speed exponent $\alpha$ depends on the cylinder slenderness. From these results the following equation is obtained and used in the prediction model to represent the dependence of the speed exponent on the cylinder slenderness $B / D$

$$
\alpha_{i}= \begin{cases}5.52, & \text { if } B / D<0.5 \\ -5.52 \log _{10}(B / D)^{2}-0.06 \log _{10}(B / D)+6.0, & \text { if } 0.5 \leq B / D \leq 2 \\ 5.46, & \text { if } B / D>2\end{cases}
$$

where $\alpha_{i}$ is the speed exponent for a specific slenderness $B / D$ and $\alpha_{0}=6$ is the speed exponent for a square cylinder $(B / D=1)$.

\subsection{Effect of the yaw angle}

When a cylinder is inclined with respect to the incoming flow direction, the frequency and amplitude of the vortex shedding noise is modified, this being dependent on the yaw angle $\beta$, defined here as the angle between the cylinder 
axis and the line perpendicular to the flow direction. Hence, a yaw angle of $0^{\circ}$ corresponds to a cross flow, whereas at $90^{\circ}$ the cylinder is aligned with the flow.

According to the independence principle it may be assumed that the incident flow speed for an inclined cylinder is the component of the freestream flow velocity $\mathbf{U}_{\infty}$ perpendicular to the yawed cylinder centreline $U_{n}=U_{\infty} \cos (\beta)$ [48]. According to the experimental results of Latorre Iglesias et al. [22] the factor $\cos \beta$ obtained from the independence principle gives a good estimation of the variation of the vortex shedding frequency with the yaw angle. This factor is used in the present model to account for the variation of the Strouhal number with the yaw angle.

$$
S t_{i} / S t_{0}=\cos \beta
$$

According to the independence principle, the dependence of the SPL on the yaw angle can be approximated using a factor of $10 \log _{10}(\cos \beta)^{\gamma}$ with $\gamma=6$. However, Latorre Iglesias et al. [22] found that different values of $\gamma$ provide a better fit with experimental results for different cylinder cross-sections. From these results the variation of the amplitude factor with the yaw angle is included in the prediction model as:

$$
\begin{cases}\eta_{i} / \eta_{0}=(\cos \beta)^{4.1}, & \text { for circular cylinders } \\ \eta_{i} / \eta_{0}=(\cos \beta)^{4.9}, & \text { for square cylinders }\end{cases}
$$

Due to a lack of data, the relation for circular cylinders is also used for elliptical cylinders, while that obtained for square cylinders is also used for rectangular cylinders.

The results obtained by Latorre Iglesias and Thompson in [43] show that, for circular, square and rectangular cylinders, values of the speed exponent $\alpha$ around 6 are obtained for the vortex shedding noise at most yaw angles. The results for elliptical cylinders showed a big variability without a clear trend. For this reason the same value of $\alpha$ is used for all yaw angles.

According to the results found in [22] the relative bandwidth for the circular and square cylinders increases with the yaw angle. From these results the following equation is obtained to account for the variation of the relative bandwidth with the yaw angle.

$$
B_{f_{i}} / B_{f_{0}}=4.5-3.5 \cos (\beta)
$$

Latorre Iglesias et al. [22] found that the increase of the relative bandwidth with the yaw angle for the rectangular cylinders with slenderness ratio 0.75 and 1.33 was similar to that for the square cylinder. Equation (25) is therefore also used for rectangular cylinders. For elliptical cylinders the relative bandwidth is considerably larger than for a circular cylinder. However, this appears to remain constant with the yaw angle, as shown in the results from [22] for cylinder eccentricities 0.63 and 0.75 . In the prediction model the relative bandwidth of an elliptical cylinder is therefore not modified by the yaw angle.

\subsection{Effects on broadband noise}

The effect of different factors on the broadband noise should be included in the prediction model. In Figures 6(b) and 7(b) it is shown that for small cylinder aspect ratio, for which the vortex shedding is not triggered, the broadband noise does not increase with the aspect ratio. Based on that, the broadband noise is assumed to be independent of the cylinder aspect ratio. The results presented in [43] show that the difference between the peak and broadband noise is nearly contant with the slenderness ratio (variations less than $3 \mathrm{~dB}$ ). On the basis of that, in the prediction model the broadband noise is assumed to be independent of the slenderness ratio of rectangular cylinders. This difference is larger for elliptical cylinders. However, only measurements with two different cylinder eccentricities were available so this is not enough to obtain conclusive results. To simplify things, it is also assumed that the broadband noise is independent of the eccentricity of elliptical cylinders. The broadband noise is also assumed to be independent on the incoming turbulence. Further research is needed to confirm or improve these assumptions.

Nevertheless, the amplitude of the broadband noise was found to be dependent on the cylinder yaw angle. To account for the variation of the broadband noise with the yaw angle, this is obtained in terms of the variation of the peak noise, as shown in Figure 12 for circular and square cylinders yawed by different angles. According to these results, in the prediction model it is supposed that the decrease of the broadband noise is directly linked to 
the reduction of the peak amplitude. Based on the results in Figure 12 the variation of the amplitude factor for the broadband noise is given by:

$$
\eta_{b_{i}} / \eta_{b_{0}}=\left(\eta_{p_{i}} / \eta_{p_{0}}\right)^{0.55}
$$

where the subscript $i$ stands for the value of the parameter after applying the correction and the subscript 0 stands for the value before applying the correction.

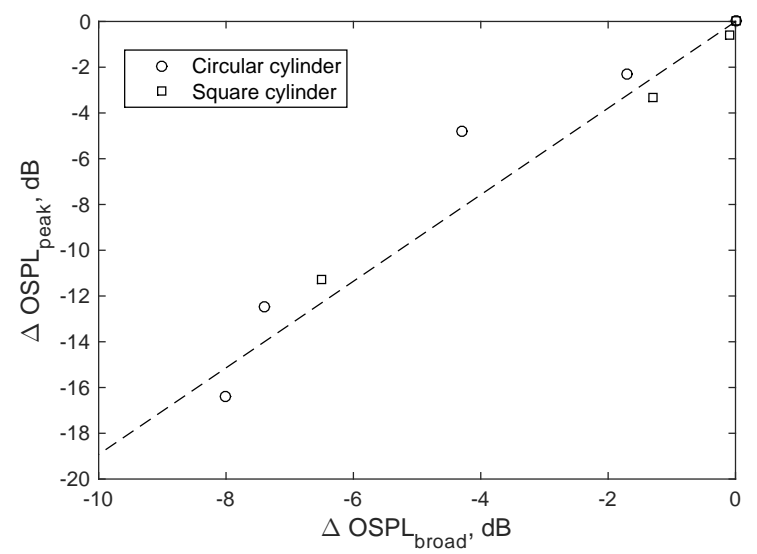

Figure 12: Relation between the decrease of the peak and broadband noise due to the increase of the yaw angle of a circular and a square cylinder [43].

\subsection{Effect of rounding the edges}

If the edges of a square cylinder are rounded, the Strouhal number and noise radiation will be modified, this change being dependent on the radius of the rounded edge $r$. Figure 13(a) shows the dependence of the Strouhal number for square cylinders with rounded edges on the radius of the rounded edge $r$, normalised by the cylinder characteristic dimension, $D$. The Strouhal number is normalised by the value for a square cylinder with sharp edges $(r=0)$. The results are fitted by least squares using a quadratic function of $\log _{10}(r / D)$ giving:

$$
S t_{i} / S t_{0}=0.15\left(\log _{10}(r / D)\right)^{2}+0.57 \log _{10}(r / D)+1.532 \text {, for } r / D>0.01
$$

Figure 13(b) shows the dependence of the SPL on the normalised radius. The effect is introduced in the prediction model as follows

$$
\eta_{i} / \eta_{0}= \begin{cases}1, & \text { if } r / D \leq 0.047 \\ 23.3 \times 10^{-3}(r / D)^{-1.2}, & \text { if } 0.047<r / D \leq 0.25 \\ 0.11, & \text { if } r / D>0.25\end{cases}
$$

Due to the lack of available data it is assumed that the speed exponent $\alpha=6$, obtained for the cases $r / D=0$ (square cylinder) and $r / D=1$ (circular cylinder), remains constant with the radius of the rounded edges. In the case of the variation of the relative bandwidth with $r$ a linear dependence is assumed from $B_{f} / f_{0}=0.28$ obtained for a square cylinder to $B_{f} / f_{0}=0.16$ obtained for the circular cylinder.

$$
B_{f_{i}} / B_{f_{0}}=-0.12(r / D)+0.28
$$

It is also assumed that these results can be applied for rectangular cylinders. 

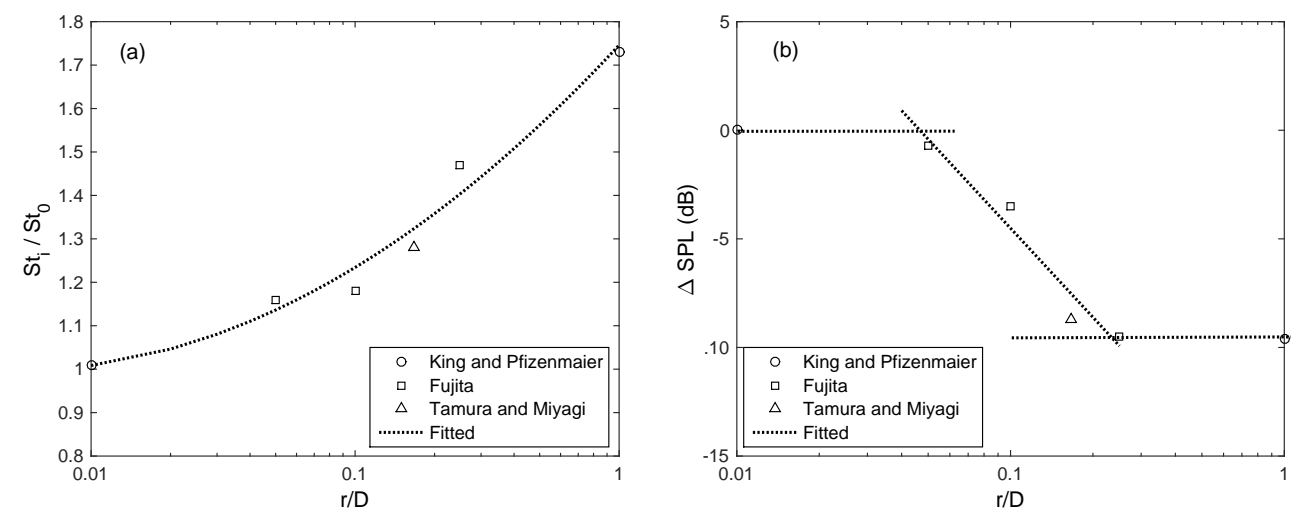

Figure 13: Dependence of the Strouhal number and SPL of a square cylinder on the radius of the rounded edge normalised by the cylinder characteristic dimension, $r / D$. Results obtained by Fujita [51], King and Pfizenmaier [20] and Tamura and Miyagi [52]. (a) Dependence of the ratio between the Strouhal number for a square cylinder with rounded edges and with sharp edges, $S t_{i} / S t_{0}$, on the normalised radius of the rounded edges. (b) Dependence of the SPL on $r / D$ for square cylinders with rounded edges.

\subsection{Effect of incoming turbulence}

In all the cases presented in the previous sections a smooth incoming flow (i.e. with low turbulence intensity) was assumed. Turbulent inflow can be relevant for pantograph noise in two situations: for the lower parts of the pantograph, which can be located inside the turbulent boundary layer developed on the roof of the train; and for struts placed in the wake of an upstream strut, at sufficient distance to neglect any flow interaction effects.

The freestream turbulence can be characterized by the turbulence intensity and the length scales. Both terms are three dimensional so they can be defined for each of the three normal directions relative to the flow. For example, the turbulence intensity in the streamwise direction can be defined as $I_{u}=u^{\prime} / U_{\infty}$, where $u^{\prime}$ is the rms fluctuating velocity component in the streamwise direction and $U_{\infty}$ is the mean flow speed. Taylor defined a turbulence parameter [53]

$$
T_{u}=\frac{u^{\prime}}{U_{\infty}}\left(\frac{\Lambda_{u}}{D}\right)^{-0.2}
$$

to show the dependency of the critical Reynolds number (that at which the transition occurs) on the turbulence intensity $\left(I_{u}\right)$ and the size of the length scale $\Lambda_{u}$ compared with the diameter of the circular cylinder $D$. Namakura et al. $[54,44,55]$ carried out several studies into the effect of incoming turbulence on the vortex shedding process undergone by bluff bodies in an air flow (e.g. square or rectangular cylinders). They found that the length scales of the incoming turbulence that have a significant effect are those comparable to the shear-layer thickness and to the cylinder characteristic dimension $D$. They found that the effect of the incoming turbulence is negligible when the turbulence length scale is significantly larger than the cylinder characteristic dimension.

In the present study only the effect of turbulence length scales comparable to the cylinder characteristic dimension is assessed. Moreover, the incoming turbulence is considered to be isotropic even if this case is ideal and unlikely to be the case for a train pantograph. The effect of the incoming turbulence on the broadband noise is not considered in the prediction model.

\subsubsection{Circular cylinders}

The effects of the incoming turbulence on the critical Reynolds number and on the Strouhal number, fluctuating lift coefficient and correlation length have been accounted for in the prediction model. First, it is assumed that the values of $S t, C_{L r m s}$ and $l_{c}$ for Reynolds numbers in the subcritical regime are independent of the incoming turbulence. However, the value of the critical Reynolds number $R e_{c}$ varies with the incoming turbulence. Figure 14(a) shows the dependence of the critical Reynolds number on Taylor's parameter according to the results found in the literature.

For Reynolds numbers higher than $R e_{c}$ it is assumed that the effect of the incoming turbulence on $S t$ is not significant so the same model as for a smooth incoming flow in the critical regime is applied, as shown in Figure 9 . In 
terms of the SPL the variation of $C_{L r m s}$ with the incoming turbulence is shown in Figure 14(b) while the variation of $l_{c}$ with the turbulence intensity is assumed to be small. Hence the variation of $\eta$ with the turbulence intensity is only dependent on $C_{L r m s}$ and this is applied additionally to that shown in Figure 9 for the critical regime in smooth flow.
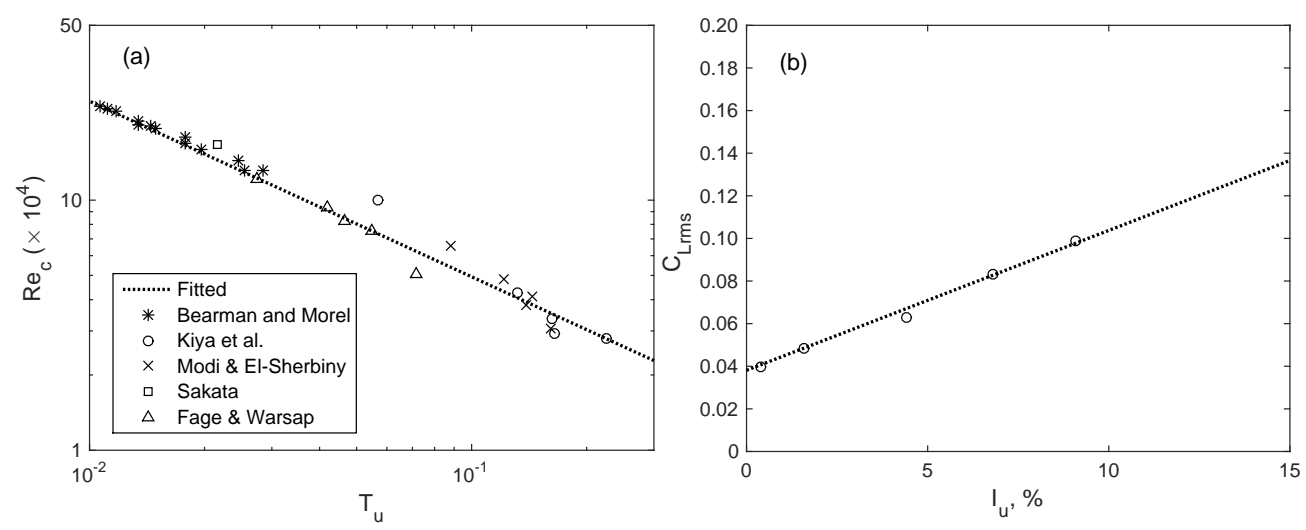

Figure 14: Effect of the incoming turbulence on the critical Reynolds number and the rms fluctuating lift coefficient. (a) Variation of the critical Reynolds number $R e_{c}$ with Taylor's parameter. (b) Variation of the rms fluctuating lift coefficient $C_{L \mathrm{rms}}$ with the turbulence intensity $I_{u}$ for Reynolds numbers in critical regime, as measured by Cheung and Melbourne [56].

In order to include in the model the effect of the incoming turbulence for circular cylinders, the following equations are used based on the fitted results shown in Figure 14.

$$
\begin{gathered}
\log _{10}\left(R e_{c}\right)=-0.702 \log _{10}\left(T_{u}\right)+4 \\
C_{L \mathrm{rms}_{i}} / C_{L \mathrm{rms}_{0}}=0.164\left(I_{u}\right)+1 \\
\eta_{i} / \eta_{0}=\left(C_{L \mathrm{rms}_{i}} / C_{L \mathrm{rms}_{0}}\right)^{2}
\end{gathered}
$$

where $I_{u}$ is expressed as a percentage. The maximum turbulence intensity considered is $15 \%$.

For elliptical cylinders, for simplicity, the same corrections are applied as for a circular cylinder independently of the cylinder eccentricity. This was not proven and differences can be expected between the critical Reynolds number and the effect of the incoming turbulence on the noise radiation between circular and elliptical cylinders.

\subsubsection{Square cylinders}

In the case of square cylinders, for the Reynolds number range of relevance to a pantograph, a change of the flow regime around the cylinder is not expected. The flow will separate at the upstream edges of the cylinder and will not be re-attached downstream. The influence of the upstream turbulence on the Strouhal number, fluctuating lift coefficient and correlation length has been assessed using the limited experimental results found in the literature.

According to these results $[52,37,57]$ the incoming turbulence does not affect the Strouhal number significantly. Hence this effect is neglected in the prediction model. However, as shown in Figure 15, the fluctuating lift coefficient and correlation length vary with the turbulence intensity of the incoming flow. The results are shown as the ratio of $C_{L r m s}$ and $l_{c}$ obtained for different inflow turbulence intensities relative to that for smooth flow. The results obtained by McLean and Gartshore [58] are included in Figure 15 together with those from Tamura and Miyagi [52] and Vickery [37]. A decrease of the fluctuating lift coefficient and the spanwise correlation length is observed with the increase of the turbulence intensity, leading to a reduction in the noise generated. This trend is the opposite to that shown in Figure 14(b) where the fluctuating lift coefficient for a circular cylinder increases with the incoming turbulence intensity. For the Reynolds numbers considered, in the case of the square cylinder, coherent vortex shedding is produced while for the circular cylinder these Reynolds numbers correspond to flow in the critical regime where the vortex shedding is disorganized and the fluctuating lift becomes more random. The different flow behaviour in both cases could be the reason for the different trend followed by the fluctuating lift with the incoming turbulence. 

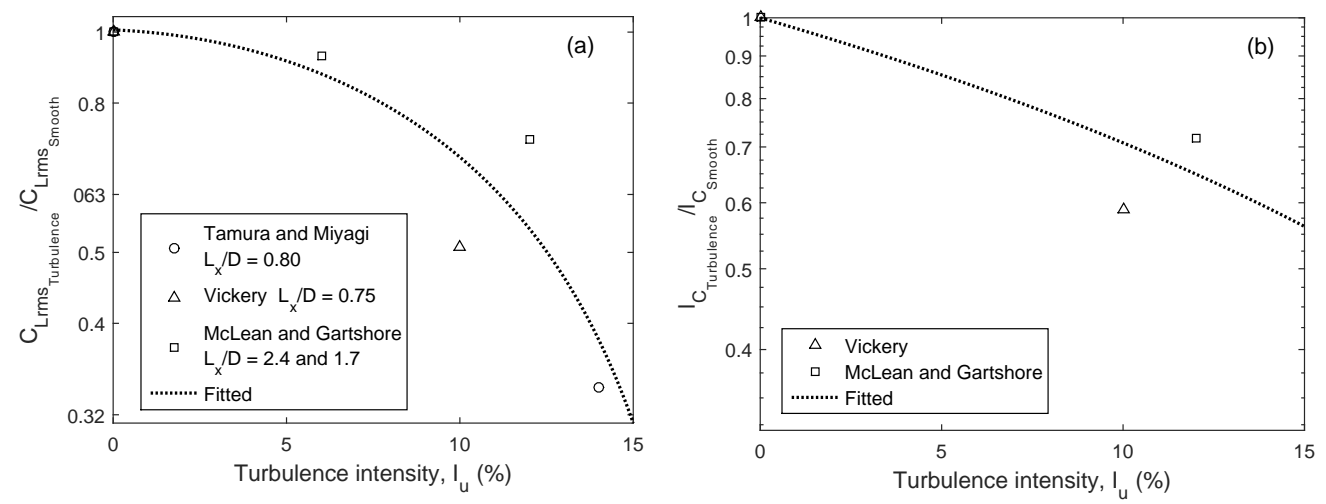

Figure 15: (a) Variation of the fluctuating lift coefficient $C_{L r m s}$ with the incoming turbulence intensity $I_{u}$ obtained by McLean and Gartshore [58], Tamura and Miyagi [52] and Vickery [37] for a square cylinder. (b) Decrease of correlation length $l_{c}$ with the incoming turbulence intensity $I_{u}$ as measured by McLean and Gartshore [58] and Vickery [37].

From the results shown in Figure 15, the following equations were obtained and used in the prediction model to account for the effect of the turbulence intensity (expressed as percentage) on the noise amplitude for a square cylinder.

$$
\begin{gathered}
C_{L i} / C_{L 0}=-2.9 \times 10^{-3} I_{u}^{2}-4.3 \times 10^{-2} I_{u}+1 \\
l_{c i} / l_{c 0}=-2.9 \times 10^{-2} I_{u}+1 \\
\eta_{i} / \eta_{0}=\left(C_{L i} / C_{L 0}\right)^{2}\left(l_{c i} / l_{c 0}\right)
\end{gathered}
$$

The maximum turbulence intensity considered is again $15 \%$.

Based on the limited results in the literature [54, 59], for rectangular cylinders with the slenderness ratios considered here $0.5 \leq B / D \leq 2.0$, it appears justified to assume the same dependence of $S t, C_{L r m s}$ and $l_{c}$ on the incoming turbulence as for square cylinders.

\section{Application of the prediction model to pantograph type DSA350}

In this section the predictions obtained using the proposed component-based model are compared with existing wind tunnel measurements of the noise radiated by a full-size pantograph type DSA350 described in Section 2. These noise measurements, carried out in the Large-scale Low-noise anechoic wind tunnel at Maibara (Japan), were provided by DB Systemtechnik [8].

The noise data were available as A-weighted noise spectra in $1 / 3$ octave bands. The pantograph was exposed to different flow speeds: 31.9, 47.2, 63.9, 77.8, 91.7, 97.2 and $111.1 \mathrm{~m} / \mathrm{s}$. The data used for this analysis were measured by a far-field microphone placed at a distance of $5 \mathrm{~m}$ normal to the flow direction from the centre of the pantograph head. Background noise corrections were applied to the data. Both knee-upstream and downstream orientations were measured. From the measured data, the spectral amplitude and shape and the speed dependence were found to be very similar between these two pantograph configurations. Therefore, only results for the knee-downstream configuration are shown here.

\subsection{Pantograph model}

Due to the complexity of the pantograph head, the flow conditions will not be the same for all the components. Because the effect of flow interaction between nearby components is not included in the prediction model, some simplifications are applied. The vortex shedding frequency $f_{0}$ for each cylinder is assumed to be the same as when they are isolated. In the pantograph head some components are assumed to be exposed to clean flow with incident flow speed equal to the free stream flow speed $U_{\infty}$, these being the upstream contact strip, horns, head lowering, springs and upstream straps and brackets. The rest of the components of the pantograph head (horn bush and downstream contact 
strip, straps and brackets) are assumed to be in the wake of the upstream contact strip. Because no measurements were available on the incident mean flow speed $U_{i}$ and turbulence intensity $I_{u}$ for each component of the pantograph head, values of $U=0.85 U_{\infty}$ and $I_{u}=10 \%$ are assumed based on results found in the literature on the flow characteristics downstream of a square cylinder [60,61] for distances equivalent to those here. In the knee region the wire arms and control strut are assumed to be in clean flow. The effect of the flow disturbances generated by the foot region on the flow around the lower parts of these cylinders was not taken into account.

Some simplifications are also made in order to include the geometry of the different components of the pantograph in the prediction model, as listed:

- Tapered struts: the dimensions of the cross-section of some of the pantograph struts change along the cylinder centreline. This is the case of the lower and upper arms and the contact strips. In these cases a constant average cross-section is assumed.

- Curved struts: the horns and the contact strips are curved. This has been accounted for in the model by dividing each cylinder into several straight segments with different orientations. This approach has the implication of reducing the aspect ratio of the cylinder as it is divided in shorter segments. This leads to a decrease in the noise level and peak frequency. This approximation was chosen as the most appropriate but further work has to be done on this effect.

- Foot regions: the wind tunnel noise tests carried out by Barsikow and King [62] using a full-scale pantograph similar to that considered here show that the noise from the foot region is broadband and significantly lower than the noise from the pantograph head. It is assumed that its contribution can be neglected.

- Yaw angles: according to the height of the catenary a pantograph will operate with yaw angles of the main struts roughly between $45^{\circ}$ and $75^{\circ}$. In the prediction model yaw angles of around $70^{\circ}$ are used according to the wind tunnel tests.

\subsection{Comparison between tests and model}

Figure 16 shows the predicted and measured noise spectra for four different flow speeds. The predicted spectral shape is similar to that measured and the same trend is seen for all the flow speeds. For frequencies between the first peak, produced by the contact strip, and the highest frequency peak, produced by the straps and springs located in the pantograph head, the agreement between measurements and predictions is good, with maximum differences of around $3 \mathrm{~dB}$. The predicted frequency of the peak radiated by the springs and straps (e.g. $4000 \mathrm{~Hz}$ at $91.7 \mathrm{~m} / \mathrm{s}$ ) is one $1 / 3$ octave band higher than the measured one. For frequencies higher than this peak the predictions are lower than the measurements by around $5 \mathrm{~dB}$. This is mainly due to the appearance of a peak in the measured noise spectra that is not obtained in the predictions, the origin of which is unknown. For frequencies below the first prominent peak the predicted noise is between 5 and $10 \mathrm{~dB}$ higher than the measured one.

Figure 17 shows the variation of the predicted and measured A-weighted OASPL as a function of the flow speed. The differences are less than $1 \mathrm{~dB}$, showing good agreement between the speed dependence of the predicted and measured data. The speed exponent is obtained from the slope of the curves obtained by using linear curve fitting in Figure 17. A value of 6.3 is obtained from the predictions and 6.5 from the tests.

Table 4 shows the contribution of each of the components of the pantograph for a flow speed of $91.7 \mathrm{~m} / \mathrm{s}$. The overall noise amplitude and the frequency of the vortex shedding peak (in 1/3 octaves) is shown for each strut. The upper and lower arms have a negligible contribution due to their large yaw angle and their elliptical cross-section. The wire arms are responsible for the peak at $1250 \mathrm{~Hz}$. The guide head and the control struts generate a peak at $315 \mathrm{~Hz}$ and a significant level of broadband noise.

The central segment of the upstream contact strip is the most significant source of noise with a large contribution of broadband noise. The straps and springs are responsible for the prominent peak at the $1 / 3$ octave of $4 \mathrm{kHz}$ and their contribution to the overall noise is also significant. Other components of the pantograph head such as the brackets and head lowering are also contributing. In the case of the horns the noisiest segment is $\mathrm{H} 2$ while the contribution from the segments $\mathrm{H} 1$ and $\mathrm{H} 3$ is lower. The contribution of the components of the knee region is almost negligible. It is shown that for pantographs with complex geometries and a large number of struts, such as here, most of the struts of the pantograph head have to be considered for an accurate prediction of the overall noise, and both peak and broadband noise should be included. 

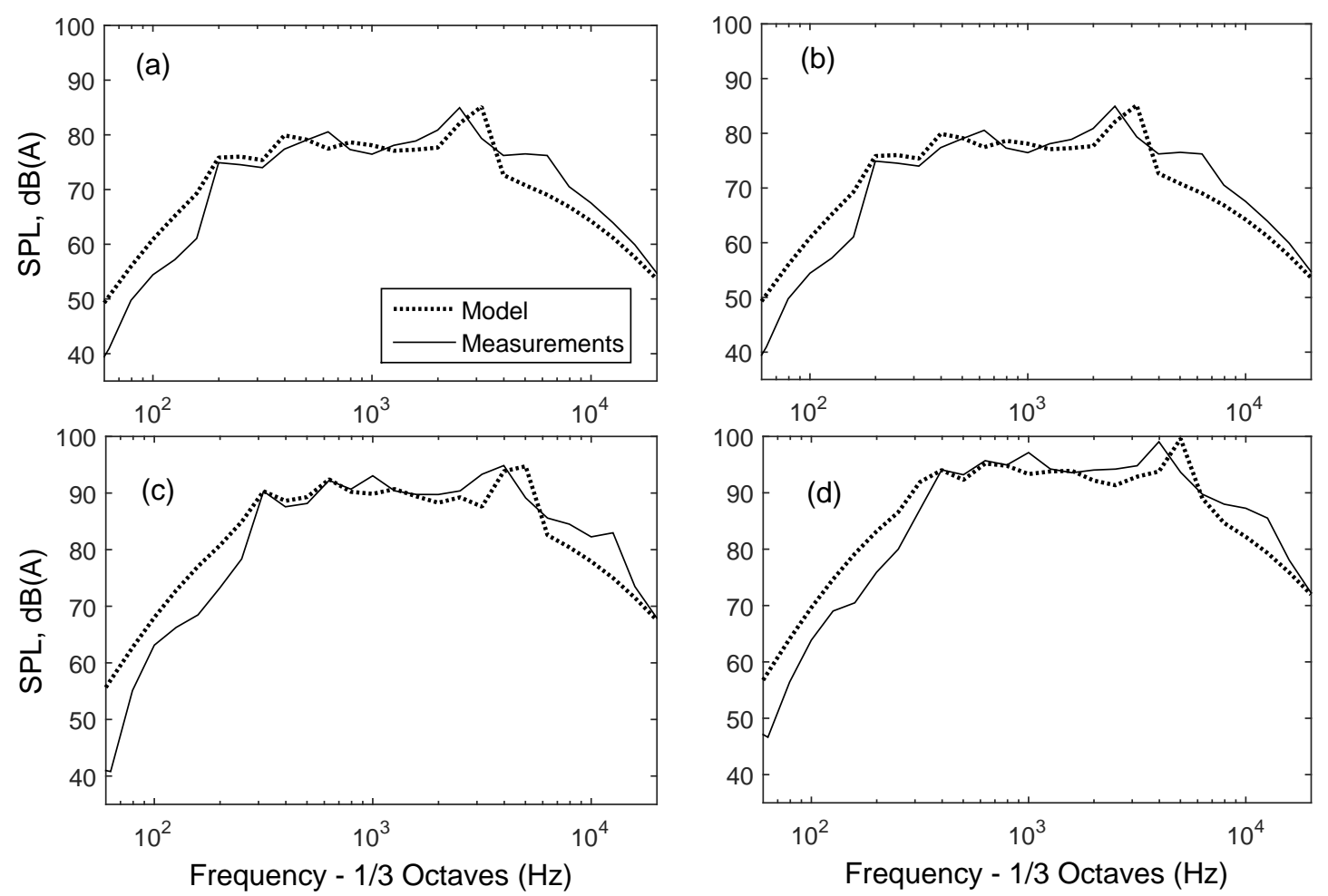

Figure 16: Predicted and measured noise spectra for different flow speeds. Pantograph type DSA350. (a) Flow speed of $63.9 \mathrm{~m} / \mathrm{s}$. (b) Flow speed of $77.8 \mathrm{~m} / \mathrm{s}$. (c) Flow speed of $97.2 \mathrm{~m} / \mathrm{s}$. (d) Flow speed of $111.1 \mathrm{~m} / \mathrm{s}$.

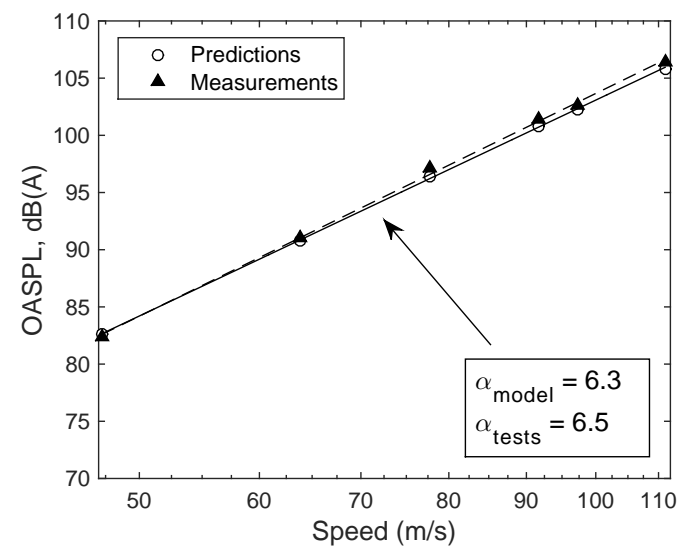

Figure 17: Variation of the predicted and measured A-weighted OASPL with the flow speed. Pantograph type DSA350.

\section{Validation of the prediction model: Japanese pantograph}

In this section the aerodynamic noise predicted using the proposed component-based model is compared with the data acquired during noise tests in the Large-scale Low-noise wind tunnel at Maibara (Japan) using a full-size Japanese pantograph. The wind tunnel tests were carried out by Manabe et al. [63, 64] for a flow speed of $20 \mathrm{~m} / \mathrm{s}$. The data was provided by RTRI. For confidentiality reasons the comparison between model and measurement is given in terms of levels with arbitrary reference.

Two different microphones were used during the experiments. Microphone M1 was located above the pantograph 
Table 4: Predicted A-weighted OASPL and vortex shedding frequency for each of the pantograph components for a flow speed of $91.7 \mathrm{~m} / \mathrm{s}$. The components (see Figure 1) are listed according with their contribution to the overall noise.

\begin{tabular}{|c|c|c|c|c|c|}
\hline Component & $\begin{array}{l}\text { OSPL } \\
\mathrm{dB}(\mathrm{A})\end{array}$ & $\begin{array}{c}f_{0} \\
\mathrm{~Hz}\end{array}$ & Component & $\begin{array}{l}\text { OSPL } \\
\mathrm{dB}(\mathrm{A})\end{array}$ & $\begin{array}{c}f_{0} \\
\mathrm{~Hz}\end{array}$ \\
\hline \multicolumn{3}{|l|}{ Head region } & Brackets down $\left(\mathrm{BK}_{d}\right)$ & 72.8 & 253 \\
\hline Contact strip 1 upstream $\left(\mathrm{CS}_{u}\right)$ & 95.8 & 373 & Contact strip 2 downstream $\left(\mathrm{CS} 2_{d}\right)$ & 71.2 & 329 \\
\hline Straps upstream $\left(\mathrm{ST}_{u}\right)$ & 94.5 & 5278 & Contact strip 3 downstream $\left(\mathrm{CS} 3_{d}\right)$ & 70.5 & 349 \\
\hline Straps downstream $\left(\mathrm{ST}_{d}\right)$ & 90.3 & 4484 & Horn bush $(\mathrm{HB})$ & 69.9 & 540 \\
\hline Head lowering $\left(\mathrm{HL}_{G} H\right)$ & 88.4 & 3145 & \multicolumn{3}{|l|}{ Knee region } \\
\hline Brakets upstream $\left(\mathrm{BK}_{u}\right)$ & 86.3 & 297 & Wire arm (WA) & 89.6 & 1442 \\
\hline Horn $2(\mathrm{H} 2)$ & 85.7 & 815 & Guide head (GH) & 87.3 & 326 \\
\hline Head lowering $(\mathrm{HL})$ & 85.5 & 563 & Control strut (CS) & 84.0 & 384 \\
\hline Contact strip 1 downstream $\left(\mathrm{CS} 1_{d}\right)$ & 84.2 & 313 & Upper arm (UA) & 70.7 & 1604 \\
\hline Contact strip 3 upstream $\left(\mathrm{CS} 3_{u}\right)$ & 83.2 & 408 & Lower arm (LA) & 46.2 & 165 \\
\hline Contact strip 2 upstream $\left(\mathrm{CS} 2_{u}\right)$ & 83.1 & 387 & \multicolumn{3}{|l|}{ Pantograph DSA350 } \\
\hline Horn $3(\mathrm{H} 3)$ & 82.1 & 677 & Overall knee region & 92 & $*$ \\
\hline Horn 1 (H1) & 81.4 & 579 & Overall head region & 100 & $*$ \\
\hline Springs (SP) & 78.0 & 4311 & Overall complete pantograph & 100 & $*$ \\
\hline
\end{tabular}

at a distance of $1.5 \mathrm{~m}$ from the pantograph head, while microphone 2 was placed at the same height as the pantograph head, $4.15 \mathrm{~m}$ downstream from it and at a lateral distance of $2 \mathrm{~m}$ from the centreline of the jet. For microphone M1 the frequency range not affected by background noise is between $50 \mathrm{~Hz}$ and $4 \mathrm{kHz}$ and for microphone M2 it is between $80 \mathrm{~Hz}$ and $5 \mathrm{kHz}$.

\subsection{Pantograph model}

The components considered for the Japanese pantograph are shown in Figure 2(a). Measurements were made with the pantograph partially assembled in three configurations:

- Foot: the components of the pantograph included in this configuration are the pantograph foot, plates and lower struts. The foot region is made of components whose shapes cannot be approximated as cylinders without assuming a significant error. For this reason, the noise from the foot region is not predicted and the measured data is used instead, this being added to the noise predicted for other pantograph struts in the other configurations.

- Struts: this includes all the components of the foot region as well as the four circular upper struts, which are yawed with respect to the incident flow by $60^{\circ}$, and the two diagonal circular struts, with yaw angle of $10^{\circ}$ separated by $433 \mathrm{~mm}$. The angle between the diagonal strut centreline and the ground is $45^{\circ}$.

- Head: this includes all the components of the foot and strut regions and also those of the pantograph head, to complete the whole pantograph: two parallel rectangular bars, one upstream of the other, a circular bar placed between and below the rectangular bars and two horns at the ends of the circular bar.

The panhead bars shown in Figure 2(b) consist of a rectangular bar with cross-section $26 \times 40 \mathrm{~mm}$ and another rectangular bar on top of it with a cross-section of about $10 \times 25 \mathrm{~mm}$. The latter is ignored in the model. From the measured data is found that the upstream panhead bar generates a peak at $80 \mathrm{~Hz}$. Considering a characteristic dimension $D=26 \mathrm{~mm}$, which in this case gives a cylinder slenderness ratio $B / D$ of $1.54(B=40 \mathrm{~mm})$, and a flow speed of $20 \mathrm{~m} / \mathrm{s}$, a Strouhal number $S t$ of 0.104 is obtained. This agrees with the Strouhal number found by Knisely [36] for rectangular cylinders with similar slenderness ratio and for similar Reynolds numbers.

The effect of the upstream strut on the flow around the struts placed downstream of it has been accounted for by reducing the incident flow speed and increasing the turbulent intensity. From the results obtained by Durao et al. [60] in the wake of a square cylinder an incident flow speed of $U_{i}=0.5 U_{\infty}$ and a turbulence intensity $I_{u}=15 \%$ are assumed for both the downstream panhead bar and the circular panhead strut. In the case of the two downstream upper struts, placed in the wake of the two upstream struts, values of $U_{i}=0.85 U_{\infty}$ and $I_{u}=5 \%$ are assumed. The value of $I_{u}$ is different to that chosen for the panhead of the pantograph type DSA350 due to the different distance and relative positions of the struts. Interaction effects are not accounted for. 
The horns present a tapered and curved geometry, as shown in Figure 2(c). Because tapered and curved cylinders are not implemented in the prediction model their geometry has been simplified. An average radius of $D=17 \mathrm{~mm}$ is obtained calculated by averaging the radius along the horn and a straight cylinder is defined joining the two ends of the horn with a length of $390 \mathrm{~mm}$. This simplification of the curved geometry is different from that applied to the horns and contact strips of the pantograph type DSA350. The approximation to a straight cylinder is preferred here because in this case the curvature is significantly larger so the horn would have to be divided into many segments with small aspect ratio, probably providing a less reliable approximation.

\subsection{Comparison between tests and model}

Figure 18 shows the comparison of the noise predicted for 'struts' configuration when the diagonal bars and upper struts are added to the noise measured for foot region. For microphone M1 the amplitude of the peak at $160 \mathrm{~Hz}$ due to the diagonal struts is well predicted although the predicted peak is narrower than the measured one. This could be due to interaction between the upstream and downstream diagonal struts, which is not included in the prediction model. The broadband noise for frequencies above $315 \mathrm{~Hz}$ is mainly produced by the foot region. The difference between the predicted and measured A-weighted OASPL is $-0.8 \mathrm{~dB}(\mathrm{~A})$. Similar results are obtained for microphone M2, with slight differences in amplitude and shedding frequency for the noise produced by the diagonal struts leading to a difference of $-1.4 \mathrm{~dB}(\mathrm{~A})$ between predictions and measurements.
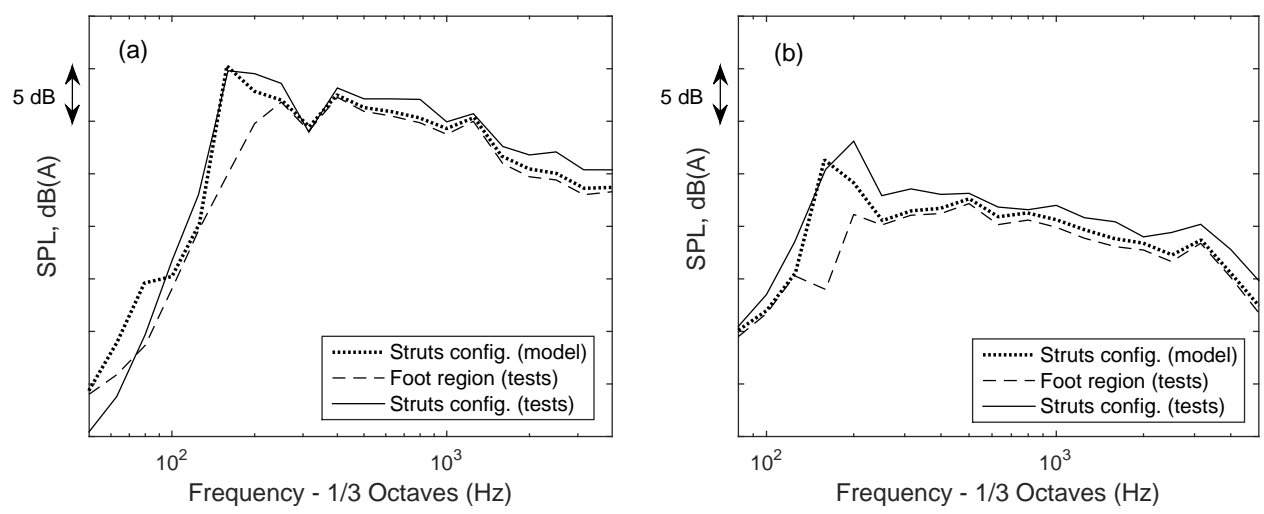

Figure 18: Comparison between predictions and measurements for the pantograph for the foot and struts configurations (pantograph head not included). (a) Microphone M1. $\triangle \mathrm{OSPL}_{\text {model-tests }}=-0.8 \mathrm{~dB}(\mathrm{~A})$. (b) Microphone M2. $\Delta \mathrm{OSPL}_{\text {model-tests }}=-1.4 \mathrm{~dB}(\mathrm{~A})$.

Figure 19 shows the results for the complete pantograph once the components of the pantograph head are added. The predicted amplitude of the peak at $80 \mathrm{~Hz}$ due to the upstream contact strip is around $5 \mathrm{~dB}$ higher than the measured one at microphone M1. In order to simplify things the contact strip was included in the prediction model as a rectangular cylinder, despite the complexity of the real geometry, as shown in Figure 2. The amplitude of the predicted vortex shedding frequency appears to be correct but the peak is significantly higher than the measured one. This could be due to the effect of the smaller rectangular cylinder acting as a rib on the larger one, decreasing the vortex shedding noise. For similar Reynolds numbers King and Barsikow [5] found that by attaching a rectangular rib along a circular cylinder, the OASPL was reduced by up to $8 \mathrm{~dB}$. Good agreement is found for microphone M2, with a difference between predicted and measured OASPL of $-1.1 \mathrm{~dB}(\mathrm{~A})$. The measured broadband noise for frequencies above $315 \mathrm{~Hz}$ is again slightly higher than that predicted.

\section{Conclusions}

A semi-empirical component-based model is proposed for estimation of the aerodynamic noise produced by a high-speed train pantograph. Each of the struts of the pantograph is approximated as a cylinder with a certain crosssection. Circular, elliptical, square and rectangular sections have been considered. The vortex shedding noise from each strut is calculated independently considering the peak and broadband noise separately. The empirical factors of 

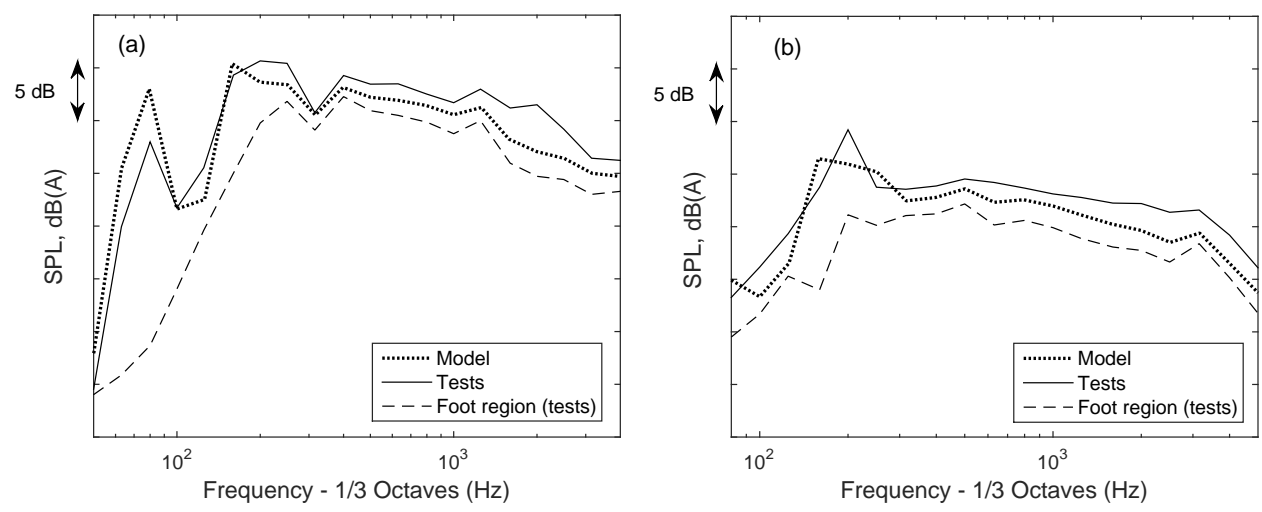

Figure 19: Comparison between predictions and measurements for the complete pantograph. (a) Microphone $\mathrm{M} 1 . \Delta \mathrm{OSPL} \mathrm{model}_{\mathrm{tests}}=-1.0 \mathrm{~dB}(\mathrm{~A})$. (b) Microphone M2. $\triangle \mathrm{OSPL}_{\text {model-tests }}=-1.1 \mathrm{~dB}(\mathrm{~A})$.

the model are chosen to fit the predictions to experimental data for individual cylinders. An experimental database is built to account for the effect of different factors on the noise from the struts: aspect ratio, flow speed, diameter, yaw angle, rounded edges, incoming turbulence and directivity. This uses both acoustic and aerodynamic parameters depending on availability. Finally the overall noise radiated by the pantograph is calculated as the incoherent sum of the noise from each of the struts.

The predictions are compared with the results of wind tunnel noise tests using two different full-scale pantographs. For a pantograph type DSA350 good agreement is found in terms of OASPL, speed dependence and spectral shape, with slight differences at some frequencies. The model allows an assessment of the contribution of each component to the overall noise. For the Japanese pantograph the predictions and measurements obtained to the side of the pantograph are in good agreement. Some differences in the noise spectrum appear for the microphone above the pantograph head due to simplifications in geometrical and flow conditions.

Some aspects would require further research such as the influence of curved and tapered cylinder geometries, interaction between nearby struts and the dependence of the broadband noise on different factors. Additionally, in most of the cases the effect on the noise of the different factors is considered independently. The interdependence of the different factors could be considered for further development of the prediction model.

Despite the simplifications and neglect of interactions, the results obtained show the potential of the proposed model. The dependence of the overall noise on modifications of the geometry and inflow conditions of any of the components of the pantograph can be assessed. This provides a useful framework to evaluate the effect of different component designs or the introduction of measures for aerodynamic noise reduction in an early stage of the design process. This prediction model can be used to complement numerical models which can be used to obtain more accurate predictions for specific cases or to increase the available database included here which is so far only based on experimental results.

\section{Acknowledgements}

The authors deeply appreciate the contribution from DB Systemtechnik and Railway Technical Research Institute (RTRI) to this work by providing the experimental data necessary to validate the prediction model.

\section{References}

[1] C. Mellet, F. Létourneaux, F. Poisson, C. Talotte, High speed train noise emission: Latest investigation of the aerodynamic/rolling noise contribution. Journal of Sound and Vibration, 293(3), (2006) 535-546. doi: 10.1016/j.jsv.2005.08.069

[2] T. Kitagawa, K. Nagakura, Aerodynamic noise generated by Shinkansen cars. Journal of Sound and Vibration, 231(3), (2000) 913-924. Aerodynamic noise generated by Shinkansen cars. doi:10.1006/jsvi.1999.2639 
[3] B. Barsikow, Experiences with various configurations of microphone arrays used to locate sound sources on railway trains operated by the DB AG. Journal of Sound and Vibration, 193(1), (1996) 283-293. doi:10.1006/jsvi.1996.0269

[4] German-French cooperation project DEUFRAKO. Annex-K - Final report, 1994.

[5] German-French Cooperation project DEUFRAKO. Annex-K2 - Final report, 1999.

[6] A. Martens, J. Wedemann, N. Meunier, A. Leclere, High speed train noise-sound source localization at fast passing trains. Proceedings of Forum Acousticum, 3rd European Congr. on Acoustics, Seville, Spain, 2002.

[7] K.G. Degen, A. Nordborg, A. Martens, J. Wedemann, L.Willenbrink, M. Bianchi, Spiral array measurements of high-speed train noise. Proceedings Inter-noise 2001, The Hague, The Netherlands, 2001.

[8] T. Lölgen, Wind tunnel noise measurements on full-scale pantograph models. The Journal of the Acoustical Society of America, 105(2) (1999) 1136. doi: 10.1121/1.425410

[9] A. Lauterbach, K. Ehrenfried, S. Loose, C. Wagner, Microphone array wind tunnel measurements of Reynolds number effects in high-speed train aeroacoustics. International Journal of Aeroacoustics 11(4) (2012) 35-62. doi: 10.1260/1475-472X.11.3-4.411

[10] H. Brick, K.R. Kirchner, K. Torsten, Noise from high-speed trains: Experimental determination of the noise radiation of the pantograph. Proceedings of Forum Acusticum 2011, Alborg, Denmark, 2011.

[11] T. Takaishi, M. Ikeda, Experimental method for wind tunnel tests to simulate turbulent flow on the roof of high-speed trains. Quarterly Report of Railway Technical Research Institute 53(3) (2012) 167-172. doi: 10.2219/rtriqr.53.167

[12] T. Sueki, M. Ikeda, T. Takaishi, T. Kurita, H. Yamada, Reduction of aerodynamic noise from high-speed pantograph using porous materials. Journal of Environment and Engineering, 5(3) (2010) 469-484. doi: 10.1299/jee.5.469

[13] M. Ikeda,M. T. Mitsumoji, T. Sueki, T. Takaishi, Aerodynamic noise reduction of a pantograph by shape-smoothing of panhead and its support and by the surface covering with porous material. Notes on Numerical Fluid Mechanics and Multidisciplinary Design, 118 (2012) 419-426. doi: 10.1007/978-4-431-53927-8_50

[14] K. Sato, R. Machida, T. Aoki, Aerodynamic sound and flow field generated from combinational inclined tapered cylinder. Open Journal of Fluid Dynamics. Open Journal of Fluid Dynamics 2(4A) (2012) 222-227. doi: 10.4236/ojfd.2012.24A025

[15] H.H. Yu, J.C. Li, H.Q. Zhang, On aerodynamic noises radiated by the pantograph system of high-speed trains. Acta Mechanica Sinica 29(3) (2013) 399-410. doi: 10.1007/s10409-013-0028-Z

[16] S. Lei, Z. Chengchun, W.G. Jing, R. Luquan, Numerical analysis of aerodynamic noise of a high-speed pantograph. Fourth International Conference on Digital Manufacturing and Automation (ICDMA), Qingdao, Shandong, China, 2013.

[17] E. Peers, Physics-based aeroacoustic modelling of bluff-bodies. PhD thesis, University of Southampton, 2009.

[18] X. Liu, D.J.Thompson, Z. Hu, V. Jurdic, Aerodynamic noise from a train pantograph. Proceedings of the Twenty-first International Congress of Sound and Vibration, Beijing, China, 2014.

[19] F.V. Hutcheson, T.F. Brooks, Noise radiation from single and multiple rod configurations. International Journal of Aeroacoustics 11(3) (2012) 291-394. doi: 10.1260/1475-472X.11.3-4.291

[20] W.F. King, E. Pfizenmaier, An experimental study of sound generated by flows around cylinders of different cross-section. Journal of Sound and Vibration 328(3) (2009) 318-337. doi:10.1016/j.jsv.2009.07.034

[21] D.J. Moreau, C.J. Doolan, Flow-induced sound of wall-mounted finite length cylinders. AIAA Journal 51(10) (2013) $2493-2502$. doi: $10.2514 / 1 . J 052391$

[22] E. Latorre Iglesias, D.J. Thompson, and M.G. Smith. Experimental study of the aerodynamic noise radiated by cylinders with different cross-sections and yaw angles. Journal of Sound and Vibration, 361 (2016) 108129. doi:10.1016/j.jsv.2015.09.044

[23] W. Behr, T. Lolgen, W. Baldauf, L. Willenbrink, R. Blaschko, K. Jager, J. Kremlacek, Low noise pantograph ASP-recent developments, Inter-noise 2000. Proceedings of the twenty-ninth International Congress on Noise Control Engineering, Nice, France, 2000.

[24] M.G. Smith, L.C. Chow, Prediction method for aerodynamic noise from aircraft landing gear. Proceedings of the fourth AIAA/CEAS Aeroacoustics Conference, Tolouse, France, 1998.

[25] Y.P Guo, Empirical prediction of aircraft landing gear noise, NASA Contractor Report 213780, 2005.

[26] D.J. Thompson, M.G. Smith, F. Coudret, Application of a component-based approach to modelling the aerodynamic noise from high-speed trains. Notes on Numerical Fluid Mechanics and Multidisciplinary Design 118 (2012) 427-435. doi:10.1007/978-4-431-53927-8_51

[27] E. Latorre Iglesias, D.J. Thompson, M.G. Smith, Component-based model for aerodynamic noise of high-speed trains. Notes on Numerical Fluid Mechanics and Multidisciplinary Design Volume 126 (2015) 481-488. doi:10.1007/978-3-662-44832-8_56

[28] D.J. Thompson, E. Latorre Iglesias, X. Liu, J. Zhu, Z. Hu. Recent developments in the prediction and control of aerodynamic noise from high-speed trains. International Journal of Rail Transportation, vol. 3, no. 3, pp. 119-150, 2015. doi:10.1080/23248378.2015.1052996

[29] M.M. Zdravkovich, Flow Around Circular Cylinders, vol. 1. Fundamentals. Oxford University Press, 1997.

[30] O.M. Phillips, The intensity of Aeolian tones. Journal of Fluid Mechanics 1(6) (1956) 607-624. doi:10.1017/S0022112056000408

[31] B. Etkin, G.K. Korbacher, R.T. Keefe, Acoustic radiation from a stationary cylinder in a fluid stream (Aeolian tones). The Journal of the Acoustical Society of America 29(1) (1957) 3036. doi:10.1121/1.1908673

[32] R.T. Keefe, Investigation of the fluctuating forces acting on a stationary circular cylinder in a subsonic stream and of the associated sound field. The Journal of the Acoustical Society of America 34(11) (1962) 1711-1714. doi:10.1121/1.1909102

[33] H. Fujita, H. Furutani, H. Suzuki, Experimental investigations and prediction of aerodynamic sound generated from square cylinders, Proceedings of the Fourth AIAA/CEAS Aeroacoustics Conference, Tolousse, France, 1998, pp. 2369

[34] A.P. Dowling, J.E. Ffowcs Williams, Sound and Sources of Sound, Horwood, Chichester, 1983.

[35] C. Norberg, Flow around a circular cylinder: aspects of fluctuating lift. Journal of Fluids and Structures 15(3) (2001) 459-469. doi:10.1006/jfls.2000.0367

[36] C.W. Knisely, Strouhal numbers of rectangular cylinders at incidence: a review and new data. Journal of Fluids and Structures 4(4) (1990) 371-393. doi:10.1016/0889-9746(90)90137-T

[37] B.J. Vickery, Fluctuating lift and drag on a long cylinder of square cross-section in a smooth and in a turbulent stream. Journal of Fluid Mechanics 25(3) (1966) 481-494. doi:10.1017/S002211206600020X

[38] G. Schewe, On the force fluctuations acting on a circular cylinder in crossflow from subcritical up to transcritical Reynolds numbers. Journal 
of Fluid Mechanics 133(1) (1983) 265-285. doi:10.1017/S0022112083001913

[39] R.H. Schlinker, M.R.Fink, R.K.Amiet, Vortex noise from non-rotating cylinders and airfoils. Proceedings of the Fourteenth Aerospace Science Meeting, Washington D.C., U.S.A., 1976, pp. 76-81.

[40] A. Okajima, Strouhal numbers of rectangular cylinders. Journal of Fluid Mechanics 123 (1982) 379-398. doi:10.1017/S0022112082003115

[41] C. Norberg, Fluctuating lift on a circular cylinder: review and new measurements, Journal of Fluids and Structures 17(1) (2003) 57-96. doi:10.1016/S0889-9746(02)00099-3

[42] J Ribeiro Duarte, Fluctuating lift and its spanwise correlation on a circular cylinder in a smooth and in a turbulent flow: a critical review. Journal of Wind Engineering and Industrial Aerodynamics 40(2) (1992) 179-198. doi:10.1016/0167-6105(92)90364-G

[43] E. Latorre Iglesias, D.J. Thompson, Wind tunnel tests on the noise radiated by cylinders with different cross-sections, Institute of Sound and Vibration Research Technical Memorandum 1002, 2014.

[44] Y. Nakamura, Y. Ohya, H. Tsuruta, Experiments on vortex shedding from flat plates with square leading and trailing edges. Journal of Fluid Mechanics 222 (1991) 437-447. doi:10.1017/S0022112091001167

[45] H. Fujita, The characteristics of the Aeolian tone radiated from two-dimensional cylinders. Fluid Dynamics Research 41(1) (2010) 015002. doi:10.1088/0169-5983/42/1/015002

[46] P. W. Bearman, T. Morel, Effect of free stream turbulence on the flow around bluff bodies. Progress in Aerospace Sciences 20(2) (1983) 97-123. doi:10.1016/0376-0421(83)90002-7

[47] W.J. Bursnall, L.K. Loftin Jr., Experimental investigation of the pressure distribution about a yawed circular cylinder in the critical Reynolds number range, DTIC Document, NASA Langley Research Centre, Technical Note 2463, 1951.

[48] M.M. Zdravkovich, Flow Around Circular Cylinders, vol. 2. Applications. Oxford University Press, 2003.

[49] V.J. Modi, E. Wiland, Unsteady aerodynamics of stationary elliptic cylinders in subcritical flow. AIAA Journal 8(10) (1969) 1814-1821. doi: $10.2514 / 3.5995$

[50] H. Fujita. The characteristics of Aeolian tone generated from elliptic and rectangular cylinders (in Japanese). Transactions of the Japan Society of Mechanical Engineers 7 (2003) 257-258.

[51] S. Yamada, H. Fujita, H. Maruta, H. Maki, J. Shiraishi, Experimental study on aerodynamic noise generated from two-dimensional models 2nd report. Effect of the angle of inclination of circular cylinders and the angle of attack of square cylinders to aerodynamic noise (in Japanese). Transactions of the Japan Society of Mechanical Engineers 63(610) (1997) 1974-1979.

[52] T. Tamura, T. Miyagi, The effect of turbulence on aerodynamic forces on a square cylinder with various corner shapes. Journal of Wind Engineering and Industrial Aerodynamics 83(1) (1999) 135-145. doi:10.1016/S0167-6105(99)00067-7

[53] G.I. Taylor, Statistical theory of turbulence. Proceedings of the Royal Society of London. Series A, Mathematical and Physical Sciences 151(873) (1935) 421-444. doi:10.1098/rspa.1935.0158

[54] Y. Nakamura, Y. Ohya, The effects of turbulence on the mean flow past two-dimensional rectangular cylinders, Journal of Fluid Mechanics 149 (1984) 255-273. doi:10.1017/S0022112084002640

[55] Y. Nakamura, Y. Ohya, S. Ozono, The effects of turbulence on bluff-body mean flow. Journal of Wind Engineering and Industrial Aerodynamics 28(1) (1988) 251-259. doi:10.1016/0167-6105(88)90121-3

[56] J.C.K. Cheung, W.H. Melbourne, Turbulence effects on some aerodynamic parameters of a circular cylinder at supercritical numbers. Journal of Wind Engineering and Industrial Aerodynamics 14(1) (1983) 399-410. doi:10.1016/0167-6105(83)90041-7

[57] B.E. Lee, The effect of turbulence on the surface pressure field of a square prism. Journal of Fluid Mechanics 69(02) (1975) 263-282. doi:10.1017/S0022112075001437

[58] I. McLean, I. Gartshore, Spanwise correlations of pressure on a rigid square section cylinder. Journal of Wind Engineering and Industrial Aerodynamics 41(1) (1992) 797-808. doi:10.1016/0167-6105(92)90498-Y

[59] H.Noda, A. Nakayama, Free-stream turbulence effects on the instantaneous pressure and forces on cylinders of rectangular cross section. Experiments in Fluids 34(4) (2003) 332-344. doi:10.1007/s00348-002-0562-0

[60] D.F.G. Durao, M.V. Heitor, J.C.F. Pereira, Measurements of turbulent and periodic flows around a square cross-section cylinder. Experiments in Fluids 6(5) (1988) 298-304. doi:10.1007/BF00538820

[61] A. Dobre, H. Hangan, Investigation of the three-dimensional intermediate wake topology for a square cylinder at high Reynolds number. Experiments in Fluids 37(4) (2004) 518-530. doi:10.1007/s00348-004-0836-9

[62] B. Barsikow, W.F. King III, Acoustical investigations of a full-scale DSA-350-SEK pantograph in an anechoic open-jet wind tunnel. DLRInterner Bericht, Deutsch-Franzsische Kooperation, Anhang K2, Technical Document 1A 6G09 T1.DZ, 1996.

[63] K. Manabe, T. Morikawa, T. Okubo, The aerodynamic sound from pantograph and the reducing method (1) - Wind tunnel tests on the existing pantograph (in Japanese). Railway Technical Research Report 1237 (1983) 655-671.

[64] K.Manabe, N. Shirakuni, T. Morikawa, Reducing method of aerodynamic noise from high speed pantograph. Transactions of the Japan Society of Mechanical Engineers Series B 51(463) (1985) 959-964. doi:10.1299/jee.5.469 